\title{
On the domain of the Nelson Hamiltonian
}

\author{
M. Griesemer and A. Wünsch \\ Fachbereich Mathematik, Universität Stuttgart, D-70569 Stuttgart, Germany
}

\begin{abstract}
The Nelson Hamiltonian is unitarily equivalent to a Hamiltonian defined through a closed, semibounded quadratic form, the unitary transformation being explicitly known and due to Gross. In this paper we study mapping properties of the Grosstransform in order to characterize regularity properties of vectors in the form domain of the Nelson Hamiltonian. Since the operator domain is a subset of the form domain, our results apply to vectors in the domain of the Hamiltonian was well. This work is a continuation of our previous work on the Fröhlich Hamiltonian.
\end{abstract}

\section{Introduction}

There is well known model, due to Nelson, describing a system of $N$ non-relativistic quantum particles (nucleons) interacting with a quantized field of scalar bosons (pions). The Hamiltonian of this model is based on a formal expression, which, in the case $N=1$, is given by

$$
-\Delta+d \Gamma(\omega)+\int v(k)\left(e^{i k x} a_{k}+e^{-i k x} a_{k}^{*}\right) d k
$$

where $\Delta$ denotes the Laplacian on $L^{2}\left(\mathbb{R}^{3}\right), d \Gamma(\omega)=\int \omega(k) a_{k}^{*} a_{k} d k$ measures the field energy, $\omega(k)=\sqrt{k^{2}+m^{2}}$ with $m \geq 0$ and $v(k)=\omega(k)^{-1 / 2}$. The integral in (1) accounts for the particle field interaction. Due to the non-square integrable decay of the form-factor $v$, the expression (1), as it stands, does not define a densely defined self-adjoint operator and its quadratic form is unbounded from below. There is a wellknown procedure to cure these problems: upon introducing an ultraviolet cutoff $|k| \leq \Lambda$ in the interaction integral, the resulting Hamiltonian $H_{\Lambda}$ conjugated with a dressing transform $U_{\Lambda}$ reveals a divergent vacuum expectation energy $-E_{\Lambda}$. When this energy is subtracted, the regularised, dressed Hamiltonian, in the limit $\Lambda \rightarrow \infty$ defines a closed and semi-bounded quadratic form and hence a self-adjoint Hamiltonian. By reversing the dressing, now using $U_{\infty}$, a self-adjoint Hamiltonian, the Nelson-Hamiltonian, is obtained [6]. By general arguments this Hamiltonian is the limit of $H_{\Lambda}+E_{\Lambda}$ as $\Lambda \rightarrow \infty$ in the norm-resolvent sense.

The procedure described above does not provide an explicit expression for the Nelson Hamiltonian, let alone its domain. It does provide, however, the form domain $D(q)$, which is given by

$$
D(q):=U_{\infty}^{*}\left(D(|p|) \cap D\left(d \Gamma(\omega)^{1 / 2}\right)\right)
$$


where $|p|=\sqrt{-\Delta}$. In this paper we study the mapping properties of $U_{\infty}^{*}$. Our main results imply that

$$
\begin{gathered}
D(q) \subset \bigcap_{0 \leq s<1} D\left(|p|^{s}\right) \cap D\left(d \Gamma(\omega)^{1 / 2}\right), \\
D(q) \cap D(|p|)=\{0\} .
\end{gathered}
$$

Since $D(H) \subset D(q)$, statements (3) and (4) hold equally for $D(H)$, and since $D\left(H_{0}^{1 / 2}\right) \subset$ $D(|p|)$ for $H_{0}:=-\Delta+d \Gamma(\omega)$, we conclude from (4) that

$$
D(H) \cap D\left(H_{0}^{1 / 2}\right)=\{0\}
$$

This means in particular that $D(H) \cap D\left(H_{0}\right)=\{0\}$.

On a heuristic level the critical exponent $s=1$ of $|p|$ in (3) and (4) can easily be understood on the basis of (2), where $U_{\infty}^{*}=\exp \left(a^{*}\left(B_{x}\right)-a\left(B_{x}\right)\right)$ and

$$
B_{x}(k)=\frac{v(k)}{\omega(k)+k^{2}} e^{i k x} \chi(|k| \geq K) \quad(K>0)
$$

For $\Psi \in D(|p|) \cap D\left(d \Gamma(\omega)^{1 / 2}\right)$ and $s \leq 1$ we expect that $U_{\infty}^{*} \Psi \in D\left(|p|^{s}\right)$ if and only if the norm of the operator $\left[|p|^{s}, B_{x}(k)\right]$ is square integrable as a function of $k$. This is true if and only if $|k|^{s} B_{x}(k)$ is square integrable, which, for the Nelson model in three dimensions, is satisfied if and only if $s<1$. The problem solved in this paper is to make this heuristic argument rigorous, the hard part being the proof of (4).

In [4] we had established results similar to (3) and (4) for the Fröhlich Hamiltonian, which corresponds to (1) with $\omega(k) \equiv 1$ and $v(k)=|k|^{-1}$. In this case (1) defines a closed, semi-bounded quadratic form and hence the procedure of Nelson is not necessary for defining a Hamiltonian, but it can still be applied. It turns out that the dressed Hamiltonian is self-adjoint on $D\left(H_{0}\right)$ and the analysis of $U_{\infty}^{*} D\left(H_{0}\right)$ is simplified by the fact that $\omega \equiv 1$. Similar remarks apply to the Nelson model in one and two space dimensions, see Section 5 and [7]. In the present paper we concentrate on the only open problem, which is the proof of (3) and (4) for a class of models, defined in terms of assumptions on $\omega$ and $v$, that is taylor made for the Nelson model in three dimensions with massive or massless bosons.

In Section 2 we describe the class of models to be considered in the ultraviolet regularized form and we introduce the corresponding class of dressing transforms. In Section 3 the construction of the Nelson Hamiltonian is given for the class of Section 2 . the abstract part of the argument being deferred to Appendix A. Section 4 is devoted to the mapping properties of $U_{\infty}^{*}$ and it contains our main results, Theorem 4.1 and Theorem 4.5. From these theorems we derive (3) and (4) for the Nelson model in Section 5 . Section 5 also describes the improved results that can be shown for the Nelson model in dimension $d \leq 2$. There are two appendices, besides Appendix A, were tools for the proofs of Section 4 are collected. 


\section{Hamiltonian with cutoff and Gross transform}

In this section we fix our notations, we define the class of regularized Hamiltonians $H_{\Lambda}$, $\Lambda<\infty$, to be considered in this paper and we introduce our assumption on $\omega$ and $v$.

Let $\mathscr{H}:=L^{2}\left(\mathbb{R}^{d}, d x\right) \otimes \mathcal{F}$ where $\mathcal{F}$ denotes the symmetric Fock space over $L^{2}\left(\mathbb{R}^{d}, d k\right)$. Through the isomorphism defined by $\varphi \otimes \eta \mapsto \varphi(x) \eta$ we may identify $\mathscr{H}$ with $L^{2}\left(\mathbb{R}^{d}, \mathcal{F}\right)$, the space of square integrable functions $x \mapsto \Psi(x) \in \mathcal{F}$ on $\mathbb{R}^{d}$. The Fourier transform of such a function will be denoted by $\Psi(p)$. As usual we use $\Psi^{(n)}$ to denote the $n$-boson component of the vector $\Psi$.

With $\mathcal{F}_{0}$ and $\mathscr{H}_{0}$ we denote the subspaces

$$
\mathcal{F}_{0}:=\bigcup_{n \geq 0} \chi(N \leq n) \mathcal{F} \text { and } \mathscr{H}_{0}:=\bigcup_{n \geq 0} \chi(N \leq n) \mathscr{H}
$$

of finite particle vectors in $\mathcal{F}$ and $\mathscr{H}$, respectively. Here in all the following $N$ denotes the number operator, which is defined by $(N \Psi)^{(n)}=n \Psi^{(n)}$.

The non-interacting system composed of particle and quantized field is described by the free Hamiltonian

$$
H_{0}:=p^{2} \otimes \mathbb{1}+\mathbb{1} \otimes d \Gamma(\omega),
$$

on $\mathscr{H}$, where $p^{2}=-\Delta$ on $L^{2}\left(\mathbb{R}^{d}\right)$ and $d \Gamma(\omega)$ denotes the second quantization of the one-particle operator given by multiplication with the dispersion relation $\omega$. That is, $(d \Gamma(\omega) \Psi)^{(0)}=0$ and for $n \geq 1$,

$$
(d \Gamma(\omega) \Psi)^{(n)}\left(k_{1}, \ldots, k_{n}\right)=\sum_{i=1}^{n} \omega\left(k_{i}\right) \Psi^{(n)}\left(k_{1}, \ldots, k_{n}\right) .
$$

On $\omega$ we will always assume that $\omega \in L_{l o c}^{\infty}\left(\mathbb{R}^{d}\right)$ and that $\omega>0$ almost everywhere. Such $\omega$ will be called "admissible". Our main results, in addition, require that

$$
(\omega) \quad \omega(k)=\omega(-k) \quad \text { and } \quad \omega(k)=O\left(k^{2}\right) \text { as }|k| \rightarrow \infty .
$$

The main examples of dispersion relations satisfying $(\omega)$ are $\omega(k) \equiv 1$ and $\omega(k)=$ $\sqrt{m^{2}+k^{2}}, m \geq 0$, which lead to the Fröhlich Hamiltonian 44 and to the Nelson model [6], respectively. The Hamiltonian $H_{0}$ is positive and self-adjoint on $D\left(H_{0}\right)=$ $D\left(p^{2} \otimes \mathbb{1}\right) \cap D(\mathbb{1} \otimes d \Gamma(\omega))$. Its form domain is given by the set $D\left(H_{0}^{1 / 2}\right)=D(|p| \otimes \mathbb{1}) \cap$ $D\left(\mathbb{1} \otimes d \Gamma(\omega)^{1 / 2}\right)$. The identity operator $\mathbb{1}$ will be omitted from now on.

The interaction of the particle and the bosonic field is given in terms of annihilation and creation of bosons. The usual annihilation and creation operators in Fock space associated with some vector $f \in L^{2}\left(\mathbb{R}^{d}\right)$ will be denoted by $a(f)$ and $a^{*}(f)$. They are closed, adjoint to each other with $D(a(f))=D\left(a^{*}(f)\right) \supset D\left(d \Gamma(\omega)^{1 / 2}\right)$ if $f \omega^{-1 / 2} \in L^{2}\left(\mathbb{R}^{d}\right)$, Lemma B.1, which means that $a(f)$ and $a^{*}(f)$ are well-defined on $D\left(H_{0}\right)$ and $D\left(H_{0}^{1 / 2}\right)$ for any admissible $\omega$. These operators obey the canonical commutation relations $\left[a(f), a^{*}(g)\right]=\langle f, g\rangle$ (others vanish), which are operator equations on $D(d \Gamma(\omega))$ if $\omega^{1 / 2} f \in L^{2}\left(\mathbb{R}^{d}\right)$, Lemma B.2. The symmetric field operators

$$
\phi(f):=a(f)+a^{*}(f), \quad \pi(f):=\phi(i f)
$$


are essentially self-adjoint on $D(d \Gamma(\omega))$ by Nelson's commutator theorem and by Lemma B.1. provided that $\omega^{1 / 2} f \in L^{2}\left(\mathbb{R}^{d}\right)$, and they satisfy the commutation relations

$$
[\phi(f), \phi(g)]=2 i \operatorname{Im}\langle f, g\rangle, \quad[\phi(f), \pi(g)]=2 i \operatorname{Re}\langle f, g\rangle,
$$

provided additionally that $f \omega^{-1 / 2} \in L^{2}\left(\mathbb{R}^{d}\right)$. The (self-adjoint) closures of the operators $\phi(f)$ and $\pi(f)$ will be denoted by the same symbols.

We will have occasion to work with generalized annihilation and creation operators $a(F)$ and $a^{*}(F)$ that are operators in $\mathscr{H}$ rather than $\mathcal{F}$. Here $F: L^{2}\left(\mathbb{R}^{d}, d x\right) \rightarrow$ $L^{2}\left(\mathbb{R}^{d}, d x\right) \otimes L^{2}\left(\mathbb{R}^{d}, d k\right)$ is a linear operator. In the simplest case $F \varphi=\varphi \otimes f$ for some $f \in L^{2}\left(\mathbb{R}^{d}, d k\right)$ and then $a^{\#}(F)=\mathbb{1} \otimes a^{\#}(f)$ is the usual annihilation or creation operator in $\mathcal{F}$. Often, but not always, the operator $F$ will be defined in terms of some function $(x, k) \mapsto F_{x}(k)$, denoted by $F$ as well, through the equation $(F \varphi)(x, k)=$ $\varphi(x) F_{x}(k)$. In this case $\left(a^{\#}(F) \Psi\right)(x)=a^{\#}\left(F_{x}\right) \Psi(x)$. Typically $F_{x}(k)=e^{-i k x} f(k)$ where $f \in L^{2}\left(\mathbb{R}^{d}\right)$ and then the operator norm of $F$ equals the norm of $f$ in $L^{2}\left(\mathbb{R}^{d}\right)$. See Appendix B of [4] for the definition of $a^{\#}(F)$ in the general case. For computations it is sometimes useful to expand $a(F)$ and $a^{*}(F)$ in terms of $a_{k}$ and $a_{k}^{*}$ by

$$
a(F)=\int\left(F(k)^{*} \otimes a_{k}\right) d k, \quad a^{*}(F)=\int\left(F(k) \otimes a_{k}^{*}\right) d k,
$$

where $F(k)$ denotes a bounded operator on the particle space $L^{2}\left(\mathbb{R}^{d}, d x\right)$ and

$$
\begin{aligned}
& \left(a_{k} \Psi\right)^{(n)}\left(x, k_{1}, \ldots, k_{n}\right)=(n+1)^{1 / 2} \Psi^{(n+1)}\left(x, k, k_{1}, \ldots, k_{n}\right) \\
& \left(a_{k}^{*} \Psi\right)^{(n)}\left(x, k_{1}, \ldots, k_{n}\right)=n^{-1 / 2} \sum_{j=1}^{n} \delta\left(k-k_{j}\right) \Psi^{(n-1)}\left(x, k_{1}, \ldots, k_{j-1}, k_{j+1}, \ldots, k_{n}\right) .
\end{aligned}
$$

The canonical commutation relations then read $\left[a_{k}, a_{k^{\prime}}^{*}\right]=\delta\left(k-k^{\prime}\right)$ and $\left[a_{k}, a_{k^{\prime}}\right]=0$. In terms of $a_{k}$ and $a_{k}^{*}$ we have

$$
\begin{aligned}
d \Gamma(\omega) & =\int \omega(k) a_{k}^{*} a_{k} d k \\
\left\|d \Gamma(\omega)^{1 / 2} \Psi\right\| & =\left(\int \omega(k)\left\|a_{k} \Psi\right\|^{2} d k\right)^{1 / 2} .
\end{aligned}
$$

We are now ready to introduce the ultraviolet regularized Hamiltonian corresponding to (1). For any $\Lambda<\infty$ we define $H_{\Lambda}: D\left(H_{0}\right) \subset \mathscr{H} \rightarrow \mathscr{H}$ by

$$
H_{\Lambda}:=H_{0}+\phi\left(G_{\Lambda}\right)
$$

where

$$
G_{\Lambda, x}(k):=v(k) e^{-i k x} \chi_{\Lambda}(k) .
$$

Here $\chi_{\Lambda}$ denotes the characteristic function of the set $\left\{k \in \mathbb{R}^{d}|| k \mid \leq \Lambda\right\}$. On the form 
factor $v: \mathbb{R}^{d} \mapsto \mathbb{C}$, we impose the conditions

$$
\begin{aligned}
& (v 1) \quad v \in L_{l o c}^{2}\left(\mathbb{R}^{d}\right) \quad \text { and } \quad v(k)=v(-k), \\
& (v 2) \quad \int_{\mathbb{R}^{d}} \frac{|v(k)|^{2}}{\left(1+k^{2}\right)^{2}} d k<\infty, \\
& (v 3) \quad \exists \sigma \geq 0: \int_{|k| \leq \sigma} \frac{|v(k)|^{2}}{\omega(k)} d k<\infty \text { and } m_{\sigma}:=\inf _{|k| \geq \sigma} \omega(k)>0,
\end{aligned}
$$

which are satisfied for example by the Nelson model, with massless or massive bosons, in three space dimensions. In one and two space dimensions, the massive Nelson model satisfies the condition $\int|v(k)|^{2}\left(1+k^{2}\right)^{-1} d k<\infty$, which is stronger then $(v 2)$ and whose consequences are discussed in Section 5. These models are therefore covered by the methods and results in [4], see also [7. Since, by $(v 1),(v 3)$, and Lemma B.1. the operator $\phi\left(G_{\Lambda}\right)(d \Gamma(\omega)+1)^{-1 / 2}$ is bounded, it follows that $\phi\left(G_{\Lambda}\right)$ is infinitesimally $H_{0}$-bounded and hence, by Kato-Rellich, $H_{\Lambda}$ is self-adjoint on $D\left(H_{0}\right)$ for all $\Lambda<\infty$. The symmetry condition in $(v 1)$ simplifies some computations, but for the main results it is inessential.

Following Nelson we now introduce a two-parameter family of unitary transformations, called Gross-transformations, by

$$
U_{K, \Lambda}=e^{i \pi\left(B_{K, \Lambda}\right)}, \quad \sigma \leq K<\Lambda \leq \infty
$$

where

$$
B_{K, \Lambda, x}(k):=-\frac{v(k)}{\omega(k)+k^{2}} e^{-i k x} \chi_{\Lambda}(k) \bar{\chi}_{K}(k)=-\frac{1}{\omega(k)+k^{2}} G_{\Lambda, x}(k) \bar{\chi}_{K}(k)
$$

and $\bar{\chi}_{K}:=1-\chi_{K}$. We will use $k B_{K, \Lambda}$ and $|k|^{s} B_{K, \Lambda}$ to denote the functions $k B_{K, \Lambda, x}(k)$ and $|k|^{s} B_{K, \Lambda, x}(k)$, respectively. The lower cutoff $K$ will be chosen sufficiently large in Lemma 3.1 and Theorem 3.3 , below. In all other results the size of $K$ is inessential and the only conditions $\sigma \leq K<\Lambda$ will not be repeated. Note that the condition $B_{K, \infty} \in L^{2}\left(\mathbb{R}^{d}, d k\right)$ is equivalent to $(v 2)$, which means that $(v 2)$ cannot be weakened. Note moreover that

$$
U_{K, \Lambda} \rightarrow U_{K, \infty} \quad(\Lambda \rightarrow \infty)
$$

strongly in $\mathscr{H}$, which follows from Lemma C.2.

Assuming $(\omega),(v 1),(v 2)$, and $(v 3)$, we can use Lemma C.1, Lemma C.3, Lemma C.7. and the identity

$$
p \cdot a^{*}\left(k B_{K, \Lambda}\right)+a\left(k B_{K, \Lambda}\right) \cdot p=a^{*}\left(k B_{K, \Lambda}\right) \cdot p+p \cdot a\left(k B_{K, \Lambda}\right)-\phi\left(k^{2} B_{K, \Lambda}\right),
$$

to find that

$$
\begin{aligned}
U_{K, \Lambda} p^{2} U_{K, \Lambda}^{*}= & p^{2}-2 a^{*}\left(k B_{K, \Lambda}\right) \cdot p-2 p \cdot a\left(k B_{K, \Lambda}\right)+2 a^{*}\left(k B_{K, \Lambda}\right) a\left(k B_{K, \Lambda}\right) \\
& +a\left(k B_{K, \Lambda}\right)^{2}+a^{*}\left(k B_{K, \Lambda}\right)^{2}+\phi\left(k^{2} B_{K, \Lambda}\right)+\left\|k B_{K, \Lambda}\right\|^{2}, \\
U_{K, \Lambda} d \Gamma(\omega) U_{K, \Lambda}^{*}= & d \Gamma(\omega)+\phi\left(\omega B_{K, \Lambda}\right)+\left\|\omega^{1 / 2} B_{K, \Lambda}\right\|^{2}, \\
U_{K, \Lambda} \phi\left(G_{\Lambda}\right) U_{K, \Lambda}^{*}= & \phi\left(G_{\Lambda}\right)+2\left\langle B_{K, \Lambda}, G_{\Lambda}\right\rangle
\end{aligned}
$$


on $D\left(H_{0}\right)$ as long as $\Lambda<\infty$. In view of $\left(\omega(k)+k^{2}\right) B_{K, \Lambda}+G_{\Lambda}=G_{K}$ the field operators $\phi(\cdot)$ in the above equations add up to $\phi\left(G_{K}\right)$. For the same reason the scalar terms add up to $E_{K}-E_{\Lambda}$ where

$$
E_{\Lambda}:=\int_{|k| \leq \Lambda} \frac{|v(k)|^{2}}{\omega(k)+k^{2}} d k .
$$

Note that $E_{\Lambda}$ may diverge as $\Lambda \rightarrow \infty$, and it does diverge for the Nelson model. Note also that there is no divergence for $|k| \rightarrow 0$ because of Assumption (v3). We therefore define

$$
\begin{aligned}
H_{K, \Lambda}^{\prime}:= & U_{K, \Lambda} H_{\Lambda} U_{K, \Lambda}^{*}+E_{\Lambda} \\
= & p^{2}+d \Gamma(\omega)+\phi\left(G_{K}\right)-2 a^{*}\left(k B_{K, \Lambda}\right) \cdot p-2 p \cdot a\left(k B_{K, \Lambda}\right) \\
& +a\left(k B_{K, \Lambda}\right)^{2}+a^{*}\left(k B_{K, \Lambda}\right)^{2}+2 a^{*}\left(k B_{K, \Lambda}\right) a\left(k B_{K, \Lambda}\right)+E_{K}
\end{aligned}
$$

which is self-adjoint on $D\left(H_{0}\right)$ for $\Lambda<\infty$ under the assumptions made in this section. Let $V_{K, \Lambda}$ denote the interaction part of $H_{K, \Lambda}^{\prime}$, so that $H_{K, \Lambda}^{\prime}=H_{0}+V_{K, \Lambda}$.

\section{Construction of the norm-resolvent limit}

In this section we describe the construction of the Nelson Hamiltonian associated with (1) in the generalized setup given by our Assumptions $(\omega),(v 1),(v 2)$, and $(v 3)$. Similar constructions given in the literature concern the special case of the Nelson Hamiltonian only [6, 1, 5, 3]. There is an abstract part in our argument that is summarized by Theorem A.1 in Appendix A. We apply this theorem to the quadratic form

$$
\begin{aligned}
W_{K, \Lambda}(\Psi):=\left\langle\Psi, V_{K, \Lambda} \Psi\right\rangle=2 \operatorname{Re}\left\{\left\langle\Psi, a\left(G_{K}\right) \Psi\right\rangle-2\left\langle a\left(k B_{K, \Lambda}\right) \Psi, p \Psi\right\rangle\right. \\
\left.+\left\|a\left(k B_{K, \Lambda}\right) \Psi\right\|^{2}+\left\langle a^{*}\left(k B_{K, \Lambda}\right) \Psi, a\left(k B_{K, \Lambda}\right) \Psi\right\rangle\right\}+E_{K}\|\Psi\|^{2}
\end{aligned}
$$

defined on $D\left(H_{0}^{1 / 2}\right)=D(|p|) \cap D\left(d \Gamma(\omega)^{1 / 2}\right)$. The subsequent Lemmas 3.1 and 3.2 verify the hypotheses of Theorem A.1, and Theorem 3.3. below, summarizes the result. Notice that much simpler and more direct characterizations of the Hamiltonian are possible under more restrictive assumptions on the decay of $v$ [4].

In the special case of the massive three-dimensional Nelson model the following two lemmas agree with Lemma 5 from [6].

Lemma 3.1. Assume $(\omega),(v 1),(v 2),(v 3)$, and let $\left\|v \bar{\chi}_{\sigma}\left(1+k^{2}\right)^{-1 / 2} \omega^{-1 / 4}\right\|<\infty$. Then, for all $\varepsilon>0$ there is a $K<\infty$ and $a b<\infty$, such that

$$
\left|W_{K, \Lambda}(\Psi)\right| \leq \varepsilon\left\|H_{0}^{1 / 2} \Psi\right\|^{2}+b\|\Psi\|^{2}
$$

for all $\Psi \in D\left(H_{0}^{1 / 2}\right)$ and all $\Lambda<\infty$.

Proof. Note that $\left\|v \bar{\chi}_{\sigma}\left(1+k^{2}\right)^{-1 / 2} \omega^{-1 / 4}\right\|<\infty$ implies $\left\|k B_{K, \infty} \omega^{-1 / 4}\right\|<\infty$, and $\left\|k B_{K, \infty} \omega^{-1 / 2}\right\|<\infty$. We estimate the terms of (7) one by one. For the first term, using Lemma B.1, we find that for every $\varepsilon>0$,

$$
\left|\left\langle\Psi, a\left(G_{K}\right) \Psi\right\rangle\right| \leq\|\Psi\|\left\|\frac{G_{K}}{\sqrt{\omega}}\right\|\left\|d \Gamma(\omega)^{1 / 2} \Psi\right\| \leq \varepsilon\left\|H_{0}^{1 / 2} \Psi\right\|^{2}+\frac{1}{4 \varepsilon}\left\|\frac{G_{K}}{\sqrt{\omega}}\right\|^{2}\|\Psi\|^{2},
$$


where $\left\|G_{K} \omega^{-1 / 2}\right\|<\infty$ by $(v 1)$ and $(v 3)$. Similarly, for the second and third terms,

$$
\left|\left\langle p \Psi, a\left(k B_{K, \Lambda}\right) \Psi\right\rangle\right| \leq\|p \Psi\|\left\|\frac{k B_{K, \Lambda}}{\sqrt{\omega}}\right\|\left\|d \Gamma(\omega)^{1 / 2} \Psi\right\| \leq \frac{1}{2}\left\|\frac{k B_{K, \infty}}{\sqrt{\omega}}\right\|\left\|H_{0}^{1 / 2} \Psi\right\|^{2},
$$

and

$$
\left\|a\left(k B_{K, \Lambda}\right) \Psi\right\|^{2} \leq\left\|\frac{k B_{K, \infty}}{\sqrt{\omega}}\right\|^{2}\left\|H_{0}^{1 / 2} \Psi\right\|^{2} .
$$

For the fourth term of $(7)$, let $N_{K}:=d \Gamma\left(\bar{\chi}_{K}\right)$ and note that for $\Psi \in D\left(H_{0}^{1 / 2}\right)$

$$
N_{K} \leq \frac{1}{m_{\sigma}} d \Gamma(\omega) .
$$

Then, with the help of Lemma B.3 and Cauchy-Schwarz,

$$
\begin{aligned}
\left|\left\langle a^{*}\left(k B_{K, \Lambda}\right) \Psi, a\left(k B_{K, \Lambda}\right) \Psi\right\rangle\right| & =\left|\left\langle\left(1+N_{K}\right)^{1 / 2} \Psi,\left(1+N_{K}\right)^{-1 / 2} a\left(k B_{K, \Lambda}\right)^{2} \Psi\right\rangle\right| \\
& \leq\left(1+\frac{1}{m_{\sigma}}\right)^{1 / 2}\left\|k B_{K, \Lambda} \omega^{-1 / 4}\right\|^{2}\left\|(1+d \Gamma(\omega))^{1 / 2} \Psi\right\|^{2} \\
& \leq\left(1+\frac{1}{m_{\sigma}}\right)^{1 / 2}\left\|k B_{K, \infty} \omega^{-1 / 4}\right\|^{2}\left(\left\|H_{0}^{1 / 2} \Psi\right\|^{2}+\|\Psi\|^{2}\right) .
\end{aligned}
$$

Since $\left\|k B_{K, \infty} \omega^{-1 / 2}\right\|$ and $\left\|k B_{K, \infty} \omega^{-1 / 4}\right\|$ become arbitrarily small for $K$ sufficiently large, the lemma is proved.

Lemma 3.2. Assume $(\omega),(v 1),(v 2),(v 3)$, and let $\left\|v \bar{\chi}_{\sigma}\left(1+k^{2}\right)^{-1 / 2} \omega^{-1 / 4}\right\|<\infty$. Then, for all $\Psi \in D\left(H_{0}^{1 / 2}\right)$, we have

$$
\left|W_{K, \Lambda_{1}}(\Psi)-W_{K, \Lambda_{2}}(\Psi)\right| \leq C_{K, \Lambda_{1}, \Lambda_{2}}\left\|\left(H_{0}+1\right)^{1 / 2} \Psi\right\|^{2}
$$

where $C_{K, \Lambda_{1}, \Lambda_{2}} \rightarrow 0$ as $\Lambda_{1}, \Lambda_{2} \rightarrow \infty$.

Proof. Note that $\left\|v \bar{\chi}_{\sigma}\left(1+k^{2}\right)^{-1 / 2} \omega^{-1 / 4}\right\|<\infty$ implies $\left\|k B_{K, \infty} \omega^{-1 / 4}\right\|<\infty$, and $\left\|k B_{K, \infty} \omega^{-1 / 2}\right\|<\infty$. By definition of $W_{K, \Lambda}$ and with $N_{K}:=d \Gamma\left(\bar{\chi}_{K}\right)$,

$$
\begin{aligned}
W_{K, \Lambda_{1}}(\Psi) & -W_{K, \Lambda_{2}}(\Psi) \\
=2 \operatorname{Re}\{ & -2\left\langle p \Psi, a\left(k B_{\Lambda_{2}, \Lambda_{1}}\right) \Psi\right\rangle+\left\|a\left(k B_{K, \Lambda_{1}}\right) \Psi\right\|^{2}-\left\|a\left(k B_{K, \Lambda_{2}}\right) \Psi\right\|^{2} \\
& \left.+\left\langle\left(1+N_{K}\right)^{1 / 2} \Psi,\left(1+N_{K}\right)^{-1 / 2}\left(a\left(k B_{K, \Lambda_{1}}\right)^{2}-a\left(k B_{K, \Lambda_{2}}\right)^{2}\right) \Psi\right\rangle\right\} .
\end{aligned}
$$

We estimate term by term with the help of the arguments from the proof of Lemma 3.1 . Using Lemma B.1.

$$
\left|\left\langle p \Psi, a\left(k B_{\Lambda_{2}, \Lambda_{1}}\right) \Psi\right\rangle\right| \leq \frac{1}{2}\left\|\frac{k B_{\Lambda_{2}, \Lambda_{1}}}{\sqrt{\omega}}\right\|\left\|H_{0}^{1 / 2} \Psi\right\|^{2},
$$

and likewise,

$$
\begin{aligned}
& \left|\left\|a\left(k B_{K, \Lambda_{1}}\right) \Psi\right\|^{2}-\left\|a\left(k B_{K, \Lambda_{2}}\right) \Psi\right\|^{2}\right| \\
& =\left|\left\|a\left(k B_{K, \Lambda_{1}}\right) \Psi\right\|-\left\|a\left(k B_{K, \Lambda_{2}}\right) \Psi\right\|\right|\left(\left\|a\left(k B_{K, \Lambda_{1}}\right) \Psi\right\|+\left\|a\left(k B_{K, \Lambda_{2}}\right) \Psi\right\|\right) \\
& \leq\left\|a\left(k B_{\Lambda_{2}, \Lambda_{1}}\right) \Psi\right\|\left(\left\|a\left(k B_{K, \Lambda_{1}}\right) \Psi\right\|+\left\|a\left(k B_{K, \Lambda_{2}}\right) \Psi\right\|\right) \\
& \leq 2\left\|\frac{k B_{\Lambda_{2}, \Lambda_{1}}}{\sqrt{\omega}}\right\|\left\|\frac{k B_{K, \infty}}{\sqrt{\omega}}\right\|\left\|H_{0}^{1 / 2} \Psi\right\|^{2},
\end{aligned}
$$


where $\left\|k B_{\Lambda_{2}, \Lambda_{1}} \omega^{-1 / 2}\right\| \rightarrow 0$ as $\Lambda_{1}, \Lambda_{2} \rightarrow \infty$. Finally, using Equation (8) and Lemma B.3.

$$
\begin{aligned}
& \left|\left\langle\left(1+N_{K}\right)^{1 / 2} \Psi,\left(1+N_{K}\right)^{-1 / 2}\left(a\left(k B_{K, \Lambda_{1}}\right)^{2}-a\left(k B_{K, \Lambda_{2}}\right)^{2}\right) \Psi\right\rangle\right| \\
& \leq\left\|\left(1+N_{K}\right)^{1 / 2} \Psi\right\|\left\|\left(1+N_{K}\right)^{-1 / 2} a\left(k B_{\Lambda_{2}, \Lambda_{1}}\right) a\left(k B_{K, \Lambda_{1}}+k B_{K, \Lambda_{2}}\right) \Psi\right\| \\
& \leq\left(1+\frac{1}{m_{\sigma}}\right)^{1 / 2}\left\|\frac{k B_{\Lambda_{2}, \Lambda_{1}}}{\sqrt[4]{\omega}}\right\| \| \frac{k\left(B_{\left.K, \Lambda_{1}+B_{K, \Lambda_{2}}\right)}^{\sqrt[4]{\omega}}\|\|(1+d \Gamma(\omega))^{1 / 2} \Psi \|\right.}{\leq 2\left(1+\frac{1}{m_{\sigma}}\right)^{1 / 2}\left\|\frac{k B_{\Lambda_{2}, \Lambda_{1}}}{\sqrt[4]{\omega}}\right\|\left\|\frac{k B_{K, \infty}}{\sqrt[4]{\omega}}\right\|\left\|\left(H_{0}+1\right)^{1 / 2} \Psi\right\|^{2}}
\end{aligned}
$$

where $\left\|k B_{\Lambda_{2}, \Lambda_{1}} \omega^{-1 / 4}\right\| \rightarrow 0$ as $\Lambda_{1}, \Lambda_{2} \rightarrow \infty$.

By the two lemmas above, $W_{K, \Lambda}$ satisfies the hypotheses of Theorem A.1 for $K$ large enough. By Lemma C.2, the assumption of part (iii) of Theorem A.1 is satisfied as well. We therefore conclude:

Theorem 3.3. Assume $(\omega)$, (v1), (v2), (v3), and let $\left\|v \bar{\chi}_{\sigma}\left(1+k^{2}\right)^{-1 / 2} \omega^{-1 / 4}\right\|<\infty$. Then, for $K$ large enough, there exists a unique, self-adjoint, semi-bounded operator $H_{K, \infty}^{\prime}$ with $D\left(H_{K, \infty}^{\prime}\right) \subset D\left(H_{0}^{1 / 2}\right)$ associated to the quadratic form

$$
\left\langle H_{0}^{1 / 2} \Psi, H_{0}^{1 / 2} \Psi\right\rangle+\lim _{\Lambda \rightarrow \infty} W_{K, \Lambda}(\Psi)
$$

which is defined for all $\Psi \in D\left(H_{0}^{1 / 2}\right)$. Moreover, we have

$$
\begin{aligned}
H_{K, \Lambda}^{\prime} & \rightarrow H_{K, \infty}^{\prime} \quad(\Lambda \rightarrow \infty), \\
H_{\Lambda}+E_{\Lambda} & \rightarrow H:=U_{K, \infty}^{*} H_{K, \infty}^{\prime} U_{K, \infty} \quad(\Lambda \rightarrow \infty)
\end{aligned}
$$

in the norm-resolvent sense and $D(H) \subset U_{K, \infty}^{*} D\left(H_{0}^{1 / 2}\right)$.

\section{Regularity of domain vectors}

From Theorem 3.3, we know that $U_{K, \infty}^{*} D\left(H_{0}^{1 / 2}\right)$ is the form domain of the operator $H$ and hence $D(H) \subset U_{K, \infty}^{*} D\left(H_{0}^{1 / 2}\right)$. This section is devoted to the study of the regularity of elements $\Psi \in U_{K, \infty}^{*} D\left(H_{0}^{1 / 2}\right)$ based on the decay of $v$, or more precisely, based on the decay of $f=\left(\omega+k^{2}\right)^{-1} v$. To stress this point and to simplify the notation we formulate most results of this section as mapping properties of some unitary operator

$$
U_{f}^{*}=e^{-i \pi(F)}, \quad F_{x}(k)=f(k) e^{-i k x}
$$

under suitable assumptions on the decay of $f \in L_{\mathrm{loc}}^{2}\left(\mathbb{R}^{d}\right)$ at $|k|=\infty$. Let

$$
U_{f, \Lambda}^{*}=e^{-i \pi\left(F_{\Lambda}\right)}, \quad F_{\Lambda, x}=F_{x} \chi_{\Lambda} .
$$

Unless otherwise stated, $\omega$ is assumed to be "admissible", which means that $\omega \in$ $L_{l o c}^{\infty}\left(\mathbb{R}^{d}\right)$ and $\omega(k)>0$ almost everywhere. The main results of this section are Theorems 4.1 and 4.5 . 
Theorem 4.1. Assume that $f \in L^{2}\left(\mathbb{R}^{d}\right)$ is a function with $|k|^{s} f,|k|^{s} f \omega^{-1 / 2} \in L^{2}\left(\mathbb{R}^{d}\right)$ for some $s \in[0,1]$. Then

$$
U_{f}^{*} D\left(H_{0}^{1 / 2}\right) \subset D\left(|p|^{s}\right) .
$$

Proof. As a preparation, we record the following three facts:

1. From Lemma B.5, we know that

$$
\begin{aligned}
\left\|\left[a^{\#}(F),|p|^{s}\right](d \Gamma(\omega)+1)^{-1 / 2}\right\| & =\left\|a^{\#}\left(\left[F,|p|^{s}\right]\right)(d \Gamma(\omega)+1)^{-1 / 2}\right\| \\
& \leq \max \left\{\left\||k|^{s} f\right\|,\left\||k|^{s} f \omega^{-1 / 2}\right\|\right\} .
\end{aligned}
$$

2. $D\left(H_{0}^{1 / 2}\right) \cap \mathscr{H}_{0}$ is a core of $H_{0}^{1 / 2}$ : If $\Psi \in D\left(H_{0}^{1 / 2}\right)$ then $\Psi_{n}:=\chi(N \leq n) \Psi \in$ $D\left(H_{0}^{1 / 2}\right) \cap \mathscr{H}_{0}$ and it is straightforward to show that $\left\|H_{0}^{1 / 2}\left(\Psi-\Psi_{n}\right)\right\| \rightarrow 0$ as $n \rightarrow \infty$.

3. If $\Psi \in D\left(H_{0}^{1 / 2}\right) \cap \mathscr{H}_{0}$ then $e^{-i \pi(F) t} \Psi \in D\left(|p|^{s}\right)$ and $t \mapsto|p|^{s} e^{-i \pi(F) t} \Psi$ is realanalytic:

It is well-known that for $\Psi \in \mathscr{H}_{0}$

$$
e^{-i \pi(F) t} \Psi=\sum_{n \geq 0} \frac{(-i t)^{n}}{n !} \pi(F)^{n} \Psi
$$

where this series is absolutely convergent for all $t \in \mathbb{R}$. The proof of this fact combined with Fact 1 shows that

$$
\sum_{n \geq 0} \frac{(-i t)^{n}}{n !}|p|^{s} \pi(F)^{n} \Psi=\sum_{n \geq 0} \frac{(-i t)^{n}}{n !} \pi(F)^{n}|p|^{s} \Psi+\sum_{n \geq 0} \frac{(-i t)^{n}}{n !}\left[|p|^{s}, \pi(F)^{n}\right] \Psi
$$

is absolutely convergent for all $t \in \mathbb{R}$. By the closedness of the operator $|p|^{s}$, it follows that $e^{-i \pi(F) t} \Psi \in D\left(|p|^{s}\right)$ and that $|p|^{s} e^{-i \pi(F) t} \Psi$ is given by (9).

We are now ready to prove the theorem. For all $\Phi \in D(|p|)$ and all $\Psi \in D\left(H_{0}^{1 / 2}\right) \cap \mathscr{H}_{0}$ it follows from Facts 1 and 3 that

$$
\begin{aligned}
& \left\langle\Phi,|p|^{s} e^{-i \pi(F)} \Psi-e^{-i \pi(F)}|p|^{s} \Psi\right\rangle=\left.\left\langle\Phi, e^{-i \pi(F)(1-t)}|p|^{s} e^{-i \pi(F) t} \Psi\right\rangle\right|_{t=0} ^{t=1} \\
& =\int_{0}^{1}\left\langle\Phi, e^{-i \pi(F)(1-t)}\left[i \pi(F),|p|^{s}\right] e^{-i \pi(F) t} \Psi\right\rangle d t .
\end{aligned}
$$

We write this in the form

$$
\left\langle|p|^{s} \Phi, e^{-i \pi(F)} \Psi\right\rangle=\left\langle\Phi, e^{-i \pi(F)}|p|^{s} \Psi\right\rangle+\int_{0}^{1}\left\langle\Phi, e^{-i \pi(F)(1-t)}\left[i \pi(F),|p|^{s}\right] e^{-i \pi(F) t} \Psi\right\rangle d t .
$$

For given $\Phi \in D(|p|)$ this equation extends to all $\Psi \in D\left(H_{0}^{1 / 2}\right)$. This follows from Fact 2 and the boundedness of $|p|^{s}\left(H_{0}+1\right)^{-1 / 2}$ and $\left[i \pi(F),|p|^{s}\right] e^{-i \pi(F) t}\left(H_{0}+1\right)^{-1 / 2}$ which follows from Fact 1 and Lemma C.2. Equation 10 now shows that for all $\Psi \in D\left(H_{0}^{1 / 2}\right)$

$$
\Phi \mapsto\left\langle|p|^{s} \Phi, e^{-i \pi(F)} \Psi\right\rangle
$$

is a bounded anti-linear functional defined on $D(|p|)$, which is a core of $|p|^{s}$. Since the operator $|p|^{s}$ is self-adjoint, it follows that $e^{-i \pi(F)} \Psi \in D\left(|p|^{s}\right)$. 
Lemma 4.2. If $f \in L_{\text {loc }}^{2}\left(\mathbb{R}^{d}\right)$, then for all $s \in[0,1]$ and all finite $\Lambda>0$

$$
\left\|(1+d \Gamma(\omega))^{-1 / 2}\left[|p|^{s}, U_{f, \Lambda}^{*}\right](1+d \Gamma(\omega))^{-1 / 2}\right\| \leq 2\left\||k|^{s} f \chi_{\Lambda} \omega^{-1 / 2}\right\| \cdot\left(1+\left\|\omega^{1 / 2} f \chi_{\Lambda}\right\|\right) .
$$

Remark: With only one of the factors $(1+d \Gamma(\omega))^{-1 / 2}$ on the right hand side of the inequality, a similar bound involving the norm $\left\||k|^{s} f \chi_{\Lambda}\right\|$ is true. Such a bound is not good enough for our purpose.

Proof. Let $F=F_{\Lambda}$ for notational simplicity. On $D\left(|p|^{s}\right)$ in the sense of quadratic forms we have

$$
\begin{aligned}
& |p|^{s} e^{-i \pi(F)}-e^{-i \pi(F)}|p|^{s}=\int_{0}^{1} e^{-i \pi(F)(1-t)}\left[i \pi(F),|p|^{s}\right] e^{-i \pi(F) t} d t \\
& =\int_{0}^{1} e^{-i \pi(F)(1-t)} a\left(\left[|p|^{s}, F\right]\right) e^{-i \pi(F) t} d t-\int_{0}^{1} e^{-i \pi(F)(1-t)} a^{*}\left(\left[F,|p|^{s}\right]\right) e^{-i \pi(F) t} d t .
\end{aligned}
$$

Using now Corollary C.5 and Lemma B.5 the desired estimate easily follows.

Lemma 4.3 and Corollary 4.4, below, are the main tools for the proof of Theorem 4.5 .

Lemma 4.3. Assume $f \in L_{\text {loc }}^{2}\left(\mathbb{R}^{d}\right)$ and in addition that $\omega^{1 / 2} f,|k|^{s} f \omega^{-1 / 2} \in L^{2}\left(\mathbb{R}^{d}\right)$ for some $s \in[0,1]$. Then, the following limit exists:

$$
\lim _{\Lambda \rightarrow \infty}(1+d \Gamma(\omega))^{-1 / 2}|p|^{s} U_{f, \Lambda}^{*}\left(1+H_{0}\right)^{-1 / 2} .
$$

Proof. Let $R_{\omega}:=(1+d \Gamma(\omega))^{-1 / 2}$ and for $0 \leq \Lambda_{1}, \Lambda_{2}<\infty$ let

$$
U_{f, \Lambda_{1}, \Lambda_{2}}^{*}:=e^{-i \pi\left(F_{\Lambda_{2}}-F_{\Lambda_{1}}\right)}=e^{-i \pi\left(F \chi_{\Lambda_{2}}\left(1-\chi_{\Lambda_{1}}\right)\right)} .
$$

From Lemma C.2, from the assumptions on $f$, and from the boundedness of $|p|^{s}(1+$ $\left.H_{0}\right)^{-1 / 2}$ it is clear that the limit $\lim _{\Lambda \rightarrow \infty} R_{\omega} U_{f, \Lambda}^{*}|p|^{s}\left(H_{0}+1\right)^{-1 / 2}$ exists. It remains to show that $R_{\omega}\left[|p|^{s}, U_{f, \Lambda}^{*}\right]\left(H_{0}+1\right)^{-1 / 2}$ has a limit as $\Lambda \rightarrow \infty$. In fact, we will show that

$$
\lim _{\Lambda \rightarrow \infty} R_{\omega}\left[|p|^{s}, U_{f, \Lambda}^{*}\right] R_{\omega}
$$

exists. To this end we use that $\left[\pi\left(F_{\Lambda_{1}}\right), \pi\left(F_{\Lambda_{2}}\right)\right]=0$ and hence,

$$
U_{f, \Lambda_{2}}^{*}-U_{f, \Lambda_{1}}^{*}=\left(U_{f, \Lambda_{2}}^{*} U_{f, \Lambda_{1}}-1\right) U_{f, \Lambda_{1}}^{*}=\left(U_{f, \Lambda_{1}, \Lambda_{2}}^{*}-1\right) U_{f, \Lambda_{1}}^{*} .
$$

From 12 , it follows that

$$
\begin{aligned}
& R_{\omega}\left[|p|^{s}, U_{f, \Lambda_{2}}^{*}\right] R_{\omega}-R_{\omega}\left[|p|^{s}, U_{f, \Lambda_{1}}^{*}\right] R_{\omega} \\
& =R_{\omega}\left[|p|^{s}, U_{f, \Lambda_{1}, \Lambda_{2}}^{*}\right] U_{f, \Lambda_{1}}^{*} R_{\omega}+R_{\omega}\left(U_{f, \Lambda_{1}, \Lambda_{2}}^{*}-1\right)\left[|p|^{s}, U_{f, \Lambda_{1}}^{*}\right] R_{\omega} \\
& =R_{\omega}\left[|p|^{s}, U_{f, \Lambda_{1}, \Lambda_{2}}^{*}\right] R_{\omega} \cdot R_{\omega}^{-1} U_{f, \Lambda_{1}}^{*} R_{\omega} \\
& \quad+R_{\omega}\left(U_{f, \Lambda_{1}, \Lambda_{2}}^{*}-1\right) R_{\omega}^{-1} \cdot R_{\omega}\left[|p|^{s}, U_{f, \Lambda_{1}}^{*}\right] R_{\omega} .
\end{aligned}
$$


where the identities $R_{\omega} \cdot R_{\omega}^{-1}=1=R_{\omega}^{-1} \cdot R_{\omega}$ have been inserted in the last equation. By Lemma 4.2 and by Corollary C.5.

$$
\begin{aligned}
& R_{\omega}\left[|p|^{s}, U_{f, \Lambda_{1}, \Lambda_{2}}^{*}\right] R_{\omega} \rightarrow 0 \\
& R_{\omega}\left(U_{f, \Lambda_{1}, \Lambda_{2}}^{*}-1\right) R_{\omega}^{-1} \rightarrow 0
\end{aligned}
$$

as $\Lambda_{1}, \Lambda_{2} \rightarrow \infty$, while

$$
\begin{aligned}
\sup _{\Lambda}\left\|R_{\omega}^{-1} U_{f, \Lambda}^{*} R_{\omega}\right\| & <\infty \\
\sup _{\Lambda}\left\|R_{\omega}\left[|p|^{s}, U_{f, \Lambda}^{*}\right] R_{\omega}\right\| & <\infty .
\end{aligned}
$$

From (13) to (17), it follows that the limit (11) exists.

Corollary 4.4. Suppose the hypotheses of Lemma 4.3 are satisfied for some $s \in[0,1]$, and moreover, that $U_{f}^{*} \Psi \in D\left(|p|^{s}\right)$ for some $\Psi \in D\left(H_{0}^{1 / 2}\right)$. Then,

$$
(1+d \Gamma(\omega))^{-1 / 2}|p|^{s} U_{f, \Lambda}^{*} \Psi \rightarrow(1+d \Gamma(\omega))^{-1 / 2}|p|^{s} U_{f}^{*} \Psi \quad(\Lambda \rightarrow \infty) .
$$

Proof. Let $R_{\omega}:=(1+d \Gamma(\omega))^{-1 / 2}$ and note that $R_{\omega}|p|^{s} \subset|p|^{s} R_{\omega}$. We know that $U_{f, \Lambda}^{*} \Psi \rightarrow U_{f}^{*} \Psi$ as $\Lambda \rightarrow \infty$ and that $U_{f, \Lambda}^{*} \Psi \in D\left(H_{0}^{1 / 2}\right) \subset D\left(|p|^{s}\right)$ by Lemma C.6. It follows that

$$
R_{\omega} U_{f, \Lambda}^{*} \Psi \rightarrow R_{\omega} U_{f}^{*} \Psi \quad(\Lambda \rightarrow \infty),
$$

where $\lim _{\Lambda \rightarrow \infty}|p|^{s} R_{\omega} U_{f, \Lambda}^{*} \Psi$ exists, by Lemma 4.3 . Since $|p|^{s}$ is a closed operator, we conclude that $R_{\omega} U_{f}^{*} \Psi \in D\left(|p|^{s}\right)$ and that

$$
\lim _{\Lambda \rightarrow \infty}|p|^{s} R_{\omega} U_{f, \Lambda}^{*} \Psi=|p|^{s} R_{\omega} U_{f}^{*} \Psi .
$$

The corollary now follows from $R_{\omega}|p|^{s} \subset|p|^{s} R_{\omega}$ and from the assumptions that $U_{f}^{*} \Psi \in$ $D\left(|p|^{s}\right)$.

Theorem 4.5. Assume $f, \omega^{1 / 2} f, f \omega^{-1 / 2} \in L^{2}\left(\mathbb{R}^{d}\right)$ and in addition that, for some $s \in[0,1],|k|^{s / 2} f,|k|^{s} f \omega^{-1 / 2} \in L^{2}\left(\mathbb{R}^{d}\right)$, while $|k|^{s} f \notin L^{2}\left(\mathbb{R}^{d}\right)$. Then

$$
U_{f}^{*} D\left(H_{0}^{1 / 2}\right) \cap D\left(|p|^{s}\right)=\{0\} .
$$

Proof. Let $\Psi \in D\left(H_{0}^{1 / 2}\right)$ and suppose that $U_{f}^{*} \Psi \in D\left(|p|^{s}\right)$ for some $s \in[0,1]$ for which the hypotheses of the theorem are satisfied. Then, of course, $\left\||p|^{s} U_{f}^{*} \Psi\right\|<\infty$. We will show that this is not true unless $\Psi=0$. Our proof is based on the identity

$$
\left\||p|^{s} U_{f}^{*} \Psi\right\|=\lim _{\varepsilon \rightarrow 0} \lim _{\Lambda \rightarrow \infty}\left\|(1+\varepsilon d \Gamma(\omega))^{-1 / 2}|p|^{s} U_{f, \Lambda}^{*} \Psi\right\|
$$

which follows from Corollary 4.4 and monotone convergence. Concerning the necessity of the regularization in terms of the operator $(1+\varepsilon d \Gamma(\omega))^{-1 / 2}$ see the remark following Lemma 4.2 and recall that $|k|^{s} f \notin L^{2}\left(\mathbb{R}^{d}\right)$ by assumption. We are now going to write 
the vector $(1+\varepsilon d \Gamma(\omega))^{-1 / 2}|p|^{s} U_{f, \Lambda}^{*} \Psi$ as sum of terms, where all but one have a norm that is uniformly bounded in $\varepsilon$ and $\Lambda$. We call them "good terms". The norm of the remaining term diverges unless $\Psi=0$. This will complete the proof.

Using

$$
|p|^{s} U_{f, \Lambda}^{*}=U_{f, \Lambda}^{*}|p|^{s}+\int_{0}^{1} d t U_{f, \Lambda}^{*}(1-t)\left[i \pi\left(F_{\Lambda}\right),|p|^{s}\right] U_{f, \Lambda}^{*}(t),
$$

where $U_{f, \Lambda}^{*}(t):=e^{-i \pi\left(F_{\Lambda}\right) t}$ (note that $U_{f, \Lambda}^{*}(1)=U_{f, \Lambda}^{*}$ and $i \pi\left(F_{\Lambda}\right)=a\left(F_{\Lambda}\right)-a^{*}\left(F_{\Lambda}\right)$ ), we obtain three summands:

$$
\begin{aligned}
& (1+\varepsilon d \Gamma(\omega))^{-1 / 2}|p|^{s} U_{f, \Lambda}^{*} \Psi \\
& =(1+\varepsilon d \Gamma(\omega))^{-1 / 2} U_{f, \Lambda}^{*}|p|^{s} \Psi+(1+\varepsilon d \Gamma(\omega))^{-1 / 2} \int_{0}^{1} d t U_{f, \Lambda}^{*}(1-t) a\left(\left[F_{\Lambda},|p|^{s}\right]\right) U_{f, \Lambda}^{*}(t) \Psi \\
& \quad-(1+\varepsilon d \Gamma(\omega))^{-1 / 2} \int_{0}^{1} d t U_{f, \Lambda}^{*}(1-t) a^{*}\left(\left[F_{\Lambda},|p|^{s}\right]\right) U_{f, \Lambda}^{*}(t) \Psi .
\end{aligned}
$$

The first two terms are good terms. Indeed, the norm of the first summand can directly be estimated by $\left\||p|^{s} \Psi\right\|$, which is finite. Using Lemmas B.5 and C.4 the norm of the second term can be estimated as follows

$$
\begin{aligned}
& \left\|(1+\varepsilon d \Gamma(\omega))^{-1 / 2} \int_{0}^{1} d t U_{f, \Lambda}^{*}(1-t) a\left(\left[F_{\Lambda},|p|^{s}\right]\right) U_{f, \Lambda}^{*}(t) \Psi\right\| \\
& \leq \int_{0}^{1} d t\left\|a\left(\left[F_{\Lambda},|p|^{s}\right]\right) U_{f, \Lambda}^{*}(t) \Psi\right\| \leq \int_{0}^{1} d t\left\|\frac{|k|^{s} F_{\Lambda, x}}{\sqrt{\omega}}\right\|\left\|d \Gamma(\omega)^{1 / 2} U_{f, \Lambda}^{*}(t) \Psi\right\| \\
& \leq\left\|\frac{|k|^{s} f}{\sqrt{\omega}}\right\|\left(1+\left\|\omega^{1 / 2} f\right\|\right)\left\|(1+d \Gamma(\omega))^{1 / 2} \Psi\right\|,
\end{aligned}
$$

where the supremum with respect to $t \in[0,1]$ and $\Lambda>0$ was taken in the last step.

It remains to show the divergence of the norm of the third term of $(19)$. Using that $e^{i k x}|p|^{s} e^{-i k x}=|p-k|^{s}$, this term reads

$$
(1+\varepsilon d \Gamma(\omega))^{-\frac{1}{2}} \int_{0}^{1} d t \int d k F_{\Lambda, x}(k) U_{f, \Lambda}^{*}(1-t) a_{k}^{*} A_{p, k} U_{f, \Lambda}^{*}(t) \Psi
$$

where we defined

$$
A_{p, k}:=|p-k|^{s}-|p|^{s}
$$

for short. Recall, that

$$
\left|A_{p, k}\right|=|| p-\left.k\right|^{s}-\left.|p|^{s}|\leq| k\right|^{s}
$$

for $p, k \in \mathbb{R}^{d}$ and $s \in[0,1]$, and that

$$
U_{f, \Lambda}^{*}(1-t) a_{k}^{*} U_{f, \Lambda}(1-t)=a_{k}^{*}-(1-t) \overline{F_{\Lambda, x}(k)} .
$$


Identity 22 is used now to commute $U_{f, \Lambda}^{*}(1-t)$ and $a_{k}^{*}$. Then, 20 becomes

$$
\begin{aligned}
& (1+\varepsilon d \Gamma(\omega))^{-\frac{1}{2}} \int_{0}^{1} d t \int d k F_{\Lambda, x}(k) a_{k}^{*} U_{f, \Lambda}^{*}(1-t) A_{p, k} U_{f, \Lambda}^{*}(t) \Psi \\
& -(1+\varepsilon d \Gamma(\omega))^{-\frac{1}{2}} \int_{0}^{1} d t \int d k(1-t)\left|F_{\Lambda}(k)\right|^{2} U_{f, \Lambda}^{*}(1-t) A_{p, k} U_{f, \Lambda}^{*}(t) \Psi,
\end{aligned}
$$

where we use $\left|F_{\Lambda}(k)\right|:=\left|F_{\Lambda, x}(k)\right|$ to point out, that this value does not depend on $x$ anymore. We will do the same for norms containing an $F$. The second term of $(23)$ is another good term, due to (21), its norm is bounded by

$$
\begin{aligned}
& \int_{0}^{1} d t(1-t) \int d k\left|F_{\Lambda}(k)\right|^{2}\left\|A_{p, k} U_{f, \Lambda}^{*}(t) \Psi\right\| \\
& \leq \int_{0}^{1} d t(1-t) \int d k|k|^{s}\left|F_{\Lambda}(k)\right|^{2}\left\|U_{f, \Lambda}^{*}(t) \Psi\right\| \leq\left\||k|^{s / 2} f\right\|^{2}\|\Psi\|,
\end{aligned}
$$

which is finite by the assumptions of the theorem.

We continue to analyze the first term of (23) and show its divergence. For short, define $\eta_{\Lambda, p, k}(t):=U_{f, \Lambda}^{*}(1-t) A_{p, k} U_{f, \Lambda}^{*}(t) \Psi$. We consider the squared norm of this first term of 23), write it as an inner product, commute the ladder operators, and use the pull-through formulas for $d \Gamma(\omega)$. This calculation reads

$$
\begin{aligned}
& \left\|(1+\varepsilon d \Gamma(\omega))^{-1 / 2} \int_{0}^{1} d t \int d k F_{\Lambda, x}(k) a_{k}^{*} \eta_{\Lambda, p, k}(t)\right\|^{2} \\
& =\int_{0}^{1} d t \int_{0}^{1} d t^{\prime} \int d k \int d k^{\prime} \overline{F_{\Lambda, x}(k)} F_{\Lambda, x}\left(k^{\prime}\right)\left\langle a_{k}^{*} \eta_{\Lambda, p, k}(t),(1+\varepsilon d \Gamma(\omega))^{-1} a_{k^{\prime}}^{*} \eta_{\Lambda, p, k^{\prime}}\left(t^{\prime}\right)\right\rangle \\
& =\int_{0}^{1} d t \int_{0}^{1} d t^{\prime} \int d k \int d k^{\prime} \overline{F_{\Lambda, x}(k)} F_{\Lambda, x}\left(k^{\prime}\right) \\
& \quad\left\langle a_{k^{\prime}} \eta_{\Lambda, p, k}(t),\left(1+\varepsilon\left(d \Gamma(\omega)+\omega(k)+\omega\left(k^{\prime}\right)\right)\right)^{-1} a_{k} \eta_{\Lambda, p, k^{\prime}}\left(t^{\prime}\right)\right\rangle \\
& +\int_{0}^{1} d t \int_{0}^{1} d t^{\prime} \int d k\left|F_{\Lambda}(k)\right|^{2}\left\langle\eta_{\Lambda, p, k}(t),(1+\varepsilon(d \Gamma(\omega)+\omega(k)))^{-1} \eta_{\Lambda, p, k}\left(t^{\prime}\right)\right\rangle \\
& =: \alpha+\beta,
\end{aligned}
$$

where $\alpha$ and $\beta$ denotes the two resulting summands.

The term $\alpha$ is a good term as we now show: by Cauchy-Schwarz

$$
|\alpha| \leq \int_{0}^{1} d t \int_{0}^{1} d t^{\prime} \int d k \int d k^{\prime}\left|F_{\Lambda}(k)\right|\left|F_{\Lambda}\left(k^{\prime}\right)\right|\left\|a_{k^{\prime}} \eta_{\Lambda, p, k}(t)\right\|\left\|a_{k} \eta_{\Lambda, p, k^{\prime}}\left(t^{\prime}\right)\right\| .
$$

By definition of $\eta_{\Lambda, p, k}(t)$, by (21) and by the identity

$$
U_{f, \Lambda}(t) a_{k} U_{f, \Lambda}^{*}(t)=a_{k}+t \cdot F_{\Lambda, x}(k),
$$

used twice, we obtain

$$
\begin{aligned}
\left\|a_{k^{\prime}} \eta_{\Lambda, p, k}(t)\right\| & \leq|k|^{s}\left\|a_{k^{\prime}} U_{f, \Lambda}^{*}(t) \Psi\right\|+|k|^{s}\left|F_{\Lambda}\left(k^{\prime}\right)\right|\|\Psi\| \\
& \leq|k|^{s}\left\|a_{k^{\prime}} \Psi\right\|+|k|^{s}\left|F_{\Lambda}\left(k^{\prime}\right)\right|\|\Psi\|
\end{aligned}
$$


We now multiply and divide $\left\|a_{k^{\prime}} \Psi\right\|$ by $\sqrt{\omega\left(k^{\prime}\right)}$, analogously for the momentum $k$, and insert (26) into (25). Using Cauchy-Schwarz we obtain

$$
\begin{aligned}
|\alpha| \leq\left\|\frac{|k|^{s} F_{\Lambda}}{\sqrt{\omega}}\right\|^{2}\left\|d \Gamma(\omega)^{1 / 2} \Psi\right\|^{2}+\left\||k|^{s / 2} F_{\Lambda}\right\|^{4}\|\Psi\|^{2} \\
+2\left\|\frac{|k|^{s} F_{\Lambda}}{\sqrt{\omega}}\right\|\left\||k|^{s / 2} F_{\Lambda}\right\|^{2}\left\|d \Gamma(\omega)^{1 / 2} \Psi\right\|\|\Psi\| .
\end{aligned}
$$

This remains finite in the limit $\Lambda \rightarrow \infty$ by the assumptions of the theorem. Thus, $\alpha$ from Equation (24) is a good term and the divergence has to be in the second term of (24), we called it $\beta$.

It remains to show that $\beta$ diverges. To this end, let

$$
R_{\varepsilon}(k):=(1+\varepsilon(d \Gamma(\omega)+\omega(k)))^{-1 / 2}
$$

for short. Then,

$$
\begin{aligned}
\beta & =\int d k\left|F_{\Lambda}(k)\right|^{2}\left\|R_{\varepsilon}(k) \int_{0}^{1} d t U_{f, \Lambda}^{*}(1-t) A_{p, k} U_{f, \Lambda}^{*}(t) \Psi\right\|^{2} \\
& =\int d k\left|F_{\Lambda}(k)\right|^{2}\left\|R_{\varepsilon}(k) A_{p, k} U_{f, \Lambda}^{*} \Psi+R_{\varepsilon}(k) \int_{0}^{1} d t\left[U_{f, \Lambda}^{*}(1-t), A_{p, k}\right] U_{f, \Lambda}^{*}(t) \Psi\right\|^{2} .
\end{aligned}
$$

Note, that $U_{f, \Lambda}^{*}(1-t) U_{f, \Lambda}^{*}(t)=U_{f, \Lambda}^{*}$ and, therefore, the first summand in the norm does not depend on $t$ anymore. If we define

$$
\begin{aligned}
& \beta_{1}:=\int d k\left|F_{\Lambda}(k)\right|^{2}\left\|R_{\varepsilon}(k) A_{p, k} U_{f, \Lambda}^{*} \Psi\right\|^{2}, \\
& \beta_{2}:=\int d k\left|F_{\Lambda}(k)\right|^{2}\left\|R_{\varepsilon}(k) \int_{0}^{1} d t\left[U_{f, \Lambda}^{*}(1-t), A_{p, k}\right] U_{f, \Lambda}^{*}(t) \Psi\right\|^{2},
\end{aligned}
$$

then $\beta \geq \frac{1}{2} \beta_{1}-2 \beta_{2}$. To complete the proof, we show that $\beta_{2}$ is a good term, while $\beta_{1}$ diverges.

For $\beta_{2}$, using Lemma B.6, Corollary C.5, and a representation of the commutator analogously to (18), we find

$$
\begin{aligned}
\beta_{2} & =\int d \ell\left|F_{\Lambda}(\ell)\right|^{2}\left\|R_{\varepsilon}(\ell) \int_{0}^{1} d t \int_{0}^{1} d r(1-t) U_{f, \Lambda}^{*}(1-t)\left[A_{p, \ell}, i \pi\left(F_{\Lambda}\right)\right] U_{f, \Lambda}^{*}(1-r+t r) \Psi\right\|^{2} \\
\leq & \int d \ell\left|F_{\Lambda}(\ell)\right|^{2}\left(\int_{0}^{1} d t \int_{0}^{1} d r(1-t)\left\|\pi\left(\left[A_{p, \ell}, F_{\Lambda}\right]\right) U_{f, \Lambda}^{*}(1-r+t r) \Psi\right\|\right)^{2} \\
\leq & \int d \ell\left|F_{\Lambda}(\ell)\right|^{2}\left(\int_{0}^{1} d t \int_{0}^{1} d r(1-t) \cdot 4|\ell|^{s / 2} \max \left\{\left\||k|^{s / 2} f \chi_{\Lambda}\right\|,\left\||k|^{s / 2} f \omega^{-1 / 2} \chi_{\Lambda}\right\|\right\}\right. \\
& \left.\left(1+\left\|\omega^{1 / 2} f \chi_{\Lambda}\right\|(1-r+t r)\right)\left\|(1+d \Gamma(\omega))^{1 / 2} \Psi\right\|\right)^{2} \\
& \leq 8 \int d \ell|\ell|^{s}\left|F_{\Lambda}(\ell)\right|^{2} \max \left\{\left\||k|^{s / 2} f \chi_{\Lambda}\right\|,\left\||k|^{s / 2} f \omega^{-1 / 2} \chi_{\Lambda}\right\|\right\} \\
& \left(1+\left\|\omega^{1 / 2} f \chi_{\Lambda}\right\|\right)\left\|(1+d \Gamma(\omega))^{1 / 2} \Psi\right\|^{2}
\end{aligned}
$$


This is finite by the hypotheses on $f$ and by Assumption $(\omega)$. In the last calculation, we used the integration variable $\ell$ instead of $k$ for avoiding misunderstandings in view of the application of Lemma B.6.

It remains to analyze $\beta_{1}$ from Equation (27). Using

$$
\left(|p-k|^{s}-|p|^{s}\right)^{2} \geq\left(|k|^{2 s}-4|p|^{s}|k|^{s}\right) \quad(s \in[0,1]),
$$

we obtain

$$
\begin{aligned}
\beta_{1} & =\int d k\left|F_{\Lambda}(k)\right|^{2}\left\langle R_{\varepsilon}(k) U_{f, \Lambda}^{*} \Psi, A_{p, k}^{2} R_{\varepsilon}(k) U_{f, \Lambda}^{*} \Psi\right\rangle \\
& \geq \int d k|k|^{2 s}\left|F_{\Lambda}(k)\right|^{2}\left\|R_{\varepsilon}(k) U_{f, \Lambda}^{*} \Psi\right\|^{2}-4 \int d k|k|^{s}\left|F_{\Lambda}(k)\right|^{2}\left\|R_{\varepsilon}(k)|p|^{\frac{s}{2}} U_{f, \Lambda}^{*} \Psi\right\|^{2} .
\end{aligned}
$$

The second term of 29$)$ is a good term, because, by Fatou, Corollary 4.4 , and monotone convergence

$$
\begin{aligned}
& \limsup _{\varepsilon \rightarrow 0} \limsup _{\Lambda \rightarrow \infty} \int d k|k|^{s}\left|F_{\Lambda}(k)\right|^{2}\left\|R_{\varepsilon}(k)|p|^{s / 2} U_{f, \Lambda}^{*} \Psi\right\|^{2} \\
& \leq \limsup _{\varepsilon \rightarrow 0} \int d k|k|^{s}|f(k)|^{2}\left\|R_{\varepsilon}(k)|p|^{s / 2} U_{f}^{*} \Psi\right\|^{2} \\
& \leq\left\||k|^{s / 2} f\right\|^{2}\left\||p|^{s / 2} U_{f}^{*} \Psi\right\|^{2}
\end{aligned}
$$

which is finite by our assumptions.

Finally, we look at the first term of $(29)$. For any $\Lambda_{0} \leq \Lambda$, by Fatou, Corollary 4.4 . and monotone convergence

$$
\begin{aligned}
& \liminf _{\varepsilon \rightarrow 0} \liminf _{\Lambda \rightarrow \infty} \int d k|k|^{2 s}\left|F_{\Lambda}(k)\right|^{2}\left\|R_{\varepsilon}(k) U_{f, \Lambda}^{*} \Psi\right\|^{2} \\
& \geq \liminf _{\varepsilon \rightarrow 0} \liminf _{\Lambda \rightarrow \infty} \int_{|k| \leq \Lambda_{0}} d k|k|^{2 s}|f(k)|^{2}\left\|R_{\varepsilon}(k) U_{f, \Lambda}^{*} \Psi\right\|^{2} \\
& \geq \liminf _{\varepsilon \rightarrow 0} \int_{|k| \leq \Lambda_{0}} d k|k|^{2 s}|f(k)|^{2}\left\|R_{\varepsilon}(k) U_{f}^{*} \Psi\right\|^{2} \\
& =\left\||k|^{s} f \chi_{\Lambda_{0}}\right\|^{2}\|\Psi\|^{2} .
\end{aligned}
$$

Since $\left\||k|^{s} f \chi_{\Lambda_{0}}\right\|$ diverges as $\Lambda_{0} \rightarrow \infty$, we conclude that $\|\Psi\|=0$ and the proof is complete.

\section{The Nelson model in dimensions $d \leq 3$}

We now turn to the Nelson model in space dimensions $d \leq 3$, where

$$
v(k)=\frac{1}{\sqrt{\omega(k)}} \quad \text { with } \quad \omega(k)=\sqrt{m^{2}+k^{2}} .
$$

If $d=3$, then the Hypothesis $(\omega)$ and $(v 1)-(v 3)$ are satisfied for any $m \geq 0$. By Theorem 3.3, the norm-resolvent limit $H$ of $H_{\Lambda}+E_{\Lambda}$ exists and its form domain is $U_{K, \infty}^{*} D\left(H_{0}^{1 / 2}\right)$ for $K$ sufficiently large. By Lemma C.4 we know that $U_{K, \infty}^{*} D\left(d \Gamma(\omega)^{1 / 2}\right)=$ 
$D\left(d \Gamma(\omega)^{1 / 2}\right)$ and hence $U_{K, \infty}^{*} D\left(H_{0}^{1 / 2}\right) \subset D\left(d \Gamma(\omega)^{1 / 2}\right)$. On the other hand, Theorem 4.1 shows that $U_{K, \infty}^{*} D\left(H_{0}^{1 / 2}\right) \subset D\left(|p|^{s}\right)$ for any $s<1$. This proves 3 in the introduction. Equation (4) follows from Theorem 4.5 with $s=1$.

If $d \in\{1,2\}$, then we need $m>0$ for $(v 3)$ to hold. In fact, if $m=0$ then even the regularized Hamiltonian $H_{\Lambda}$ is unbounded below. As far as $(v 2)$ is concerned, we now have the stronger property

$$
\int_{\mathbb{R}^{d}} \frac{|v(k)|^{2}}{1+k^{2}} d k<\infty
$$

It follows that $|k| B_{x}(k)$ is square-integrable for $d \leq 2$, which, by making a mild additional decay assumption allows us to prove that $D(H)=U_{\infty}^{*} D\left(H_{0}\right)$ along the line of arguments given in [4] The critical exponent $(5-d) / 2$ in following theorem can now be understood on the basis of the heuristics argument given in the introduction for the regularity preserved by the Gross transform $U_{\infty}^{*}$. A detailed proof is given in [7].

Theorem 5.1. For the massive Nelson Hamiltonian $H$ in dimensions $d \leq 2$ we have

$$
D(H) \subset \bigcap_{0 \leq s<(5-d) / 2} D\left(|p|^{s}\right) \cap D(d \Gamma(\omega))
$$

and

$$
D(H) \cap D\left(|p|^{(5-d) / 2}\right)=\{0\} .
$$

\section{A Quadratic forms and resolvent convergence}

The following theorem contains the abstract part behind Theorem 3.3. It agrees with Theorem A.1 of [4] with the exception of additional statement that is needed here. There are similar theorems by Nelson [6] and Ammari [1].

Theorem A.1. Let $H_{0} \geq 0$ be a self-adjoint operator in the Hilbert space $\mathscr{H}$ and let $\|\Psi\|_{0}:=\left\|\left(H_{0}+1\right)^{1 / 2} \Psi\right\|$ for $\Psi \in D\left(H_{0}^{1 / 2}\right)$. For each $\Lambda<\infty$ let $W_{\Lambda}$ be a quadratic form defined on $D\left(H_{0}^{1 / 2}\right)$ such that

(a) for all $\Psi \in D\left(H_{0}^{1 / 2}\right)$ and all $\Lambda<\infty$,

$$
\left|W_{\Lambda}(\Psi)\right| \leq a\|\Psi\|_{0}^{2}+b_{\Lambda}\|\Psi\|^{2}
$$

where $a<1$,

(b) for all $\Psi \in D\left(H_{0}^{1 / 2}\right)$,

$$
\left|W_{\Lambda}(\Psi)-W_{\Lambda^{\prime}}(\Psi)\right| \leq C_{\Lambda, \Lambda^{\prime}}\|\Psi\|_{0}^{2},
$$

where $C_{\Lambda, \Lambda^{\prime}} \rightarrow 0$ as $\Lambda, \Lambda^{\prime} \rightarrow \infty$.

Let $W_{\infty}(\Phi, \Psi):=\lim _{\Lambda \rightarrow \infty} W_{\Lambda}(\Phi, \Psi)$. Then, the following statements hold true: 
(i) Statement (a) extends to $\Lambda=\infty$ with some finite $b_{\infty}$, and for each $\Lambda \leq \infty$, there exists a self-adjoint, semibounded operator $H_{\Lambda}$ with $D\left(H_{\Lambda}\right) \subset D\left(H_{0}^{1 / 2}\right)$ and

$$
\left\langle\Phi, H_{\Lambda} \Psi\right\rangle=\left\langle H_{0}^{1 / 2} \Phi, H_{0}^{1 / 2} \Psi\right\rangle+W_{\Lambda}(\Phi, \Psi)
$$

for all $\Phi \in D\left(H_{0}^{1 / 2}\right)$ and $\Psi \in D\left(H_{\Lambda}\right)$.

(ii) For all $z \in \mathbb{C} \backslash \mathbb{R}$,

$$
\left(H_{\Lambda}-z\right)^{-1} \rightarrow\left(H_{\infty}-z\right)^{-1} \quad(\Lambda \rightarrow \infty)
$$

in the operator norm.

(iii) If $U_{\Lambda}, 0<\Lambda \leq \infty$, is a one-parameter family of unitary operator with $\left(H_{0}+\right.$ $1)^{-1 / 2}\left(U_{\Lambda}-U_{\infty}\right) \rightarrow 0$ as $\Lambda \rightarrow \infty$, then for all $z \in \mathbb{C} \backslash \mathbb{R}$,

$$
\left(U_{\Lambda}^{*} H_{\Lambda} U_{\Lambda}-z\right)^{-1} \rightarrow\left(U_{\infty}^{*} H_{\infty} U_{\infty}-z\right)^{-1} \quad(\Lambda \rightarrow \infty)
$$

in the operator norm.

Proof. We only prove part (iii). Parts (i) and (ii) are proven in [4]. From the first part of the theorem (and its proof) it follows that

$$
H_{0} \leq \frac{2}{1-a}\left(H_{\Lambda}+M\right), \quad \Lambda_{0} \leq \Lambda \leq \infty
$$

with constants $a \in(0,1)$ and $M>0$. This implies that $\left(H_{0}+1\right)^{1 / 2}\left(H_{\Lambda}-z\right)^{-1}$, for $z \in \mathbb{C} \backslash \mathbb{R}$, is bounded uniformly in $\Lambda \geq \Lambda_{0}$. Let $R_{\Lambda}(z):=\left(H_{\Lambda}-z\right)^{-1}$. Then, for $z \in \mathbb{C} \backslash \mathbb{R}$

$$
\begin{aligned}
& \left(U_{\Lambda}^{*} H_{\Lambda} U_{\Lambda}-z\right)^{-1}-\left(U_{\infty}^{*} H_{\infty} U_{\infty}-z\right)^{-1} \\
& \quad=U_{\Lambda}^{*} R_{\Lambda}(z) U_{\Lambda}-U_{\infty}^{*} R_{\infty}(z) U_{\infty} \\
& \quad=U_{\Lambda}^{*} R_{\Lambda}(z)\left(U_{\Lambda}-U_{\infty}\right)+U_{\Lambda}^{*}\left(R_{\Lambda}(z)-R_{\infty}(z)\right) U_{\infty}+\left(U_{\Lambda}^{*}-U_{\infty}^{*}\right) R_{\infty}(z) U_{\infty}
\end{aligned}
$$

where all three terms vanish in the limit $\Lambda \rightarrow \infty$ by the assumption on $U_{\Lambda}$ combined with the boundedness of $\left(H_{0}+1\right)^{1 / 2} R_{\Lambda}(z)$, and by the convergence $R_{\Lambda}(z) \rightarrow R_{\infty}(z)$ known from part (ii).

\section{B Creation and annihilation operators}

This section contains bounds on creation and annihilation operators relative to other, unbounded operators. Prototypical for many of the following bounds are

$$
\|a(f) \Psi\| \leq\|f\| \cdot\left\|N^{1 / 2} \Psi\right\|, \quad\left\|a^{*}(f) \Psi\right\| \leq\|f\| \cdot\left\|(N+1)^{1 / 2} \Psi\right\|,
$$

which follow immediately from the definitions of $a(f)$ and $a^{*}(f)$ and from the canonical commutation relations, see, e.g. [4]. As in main part of this paper, we will always assume that $\omega \in L_{l o c}^{\infty}\left(\mathbb{R}^{d}\right)$ and that $\omega>0$ almost everywhere. 
Lemma B.1. Let $\alpha \geq \frac{1}{2}$ and $f \in L^{2}\left(\mathbb{R}^{d}\right)$. Then, for all $\Psi \in D\left(d \Gamma(\omega)^{\alpha}\right)$,

$$
\begin{aligned}
\|a(f) \Psi\| & \leq\left\|f \omega^{-\alpha}\right\|\left\|d \Gamma(\omega)^{\alpha} \Psi\right\|, \\
\left\|a^{*}(f) \Psi\right\| & \leq \max \left\{\|f\|,\left\|f \omega^{-\alpha}\right\|\right\}\left\|(1+d \Gamma(\omega))^{\alpha} \Psi\right\| .
\end{aligned}
$$

Proof. From $a(f) \Psi=\int \overline{f(k)} a_{k} \Psi d k$ and Cauchy-Schwarz it follows that

$$
\|a(f) \Psi\|=\left\|\int \overline{f(k)} \omega(k)^{-\alpha} \omega(k)^{\alpha} a_{k} \Psi d k\right\| \leq\left\|f \omega^{-\alpha}\right\|\left\langle\Psi, d \Gamma\left(\omega^{2 \alpha}\right) \Psi\right\rangle .
$$

The condition $2 \alpha \geq 1$ implies that $d \Gamma\left(\omega^{2 \alpha}\right) \leq d \Gamma(\omega)^{2 \alpha}$ and thus, the first inequality of the lemma is proved. The second one follows from the first and from $\left\|a^{*}(f) \Psi\right\|^{2}=$ $\|a(f) \Psi\|^{2}+\|f\|^{2}\|\Psi\|^{2}$.

Lemma B.2. Suppose that $f, f \omega^{-1 / 2} \in L^{2}\left(\mathbb{R}^{d}\right)$. Then, for all $\Psi \in D(d \Gamma(\omega))$,

$$
\begin{aligned}
\left\|d \Gamma(\omega)^{1 / 2} a(f) \Psi\right\| & \leq\left\|f \omega^{-1 / 2}\right\|\|d \Gamma(\omega) \Psi\|, \\
\left\|d \Gamma(\omega)^{1 / 2} a^{*}(f) \Psi\right\| & \leq\left(\left\|\omega^{1 / 2} f\right\|+\left\|f \omega^{-1 / 2}\right\|\right)\|(1+d \Gamma(\omega)) \Psi\| .
\end{aligned}
$$

Proof. Let $\Psi \in D(d \Gamma(\omega))$ and note that $\left\|d \Gamma(\omega)^{1 / 2} a_{k} \Psi\right\| \leq\left\|(d \Gamma(\omega)+\omega(k))^{1 / 2} a_{k} \Psi\right\|=$ $\left\|a_{k} d \Gamma(\omega)^{1 / 2} \Psi\right\|$ because $\omega \geq 0$. Using this and Cauchy-Schwarz, we obtain

$$
\begin{aligned}
\left\|d \Gamma(\omega)^{1 / 2} a(f) \Psi\right\| & \leq \int d k \mid f(k)\|\| d \Gamma(\omega)^{1 / 2} a_{k} \Psi \| \\
& \leq\left(\int d k \frac{|f(k)|^{2}}{\omega(k)}\right)^{1 / 2}\left(\int d k \omega(k)\left\|a_{k} d \Gamma(\omega)^{1 / 2} \Psi\right\|\right)^{1 / 2} \\
& =\left\|\frac{f}{\sqrt{\omega}}\right\| \cdot\|d \Gamma(\omega) \Psi\|,
\end{aligned}
$$

which proves the first estimate. To prove the second one, we set $R_{\omega}:=(1+d \Gamma(\omega))^{-1}$ and we use that

$$
d \Gamma(\omega)^{1 / 2} a^{*}(f) R_{\omega}=d \Gamma(\omega)^{1 / 2} R_{\omega} a^{*}(f)-d \Gamma(\omega)^{1 / 2} R_{\omega} a^{*}(\omega f) R_{\omega}
$$

because $\left[a^{*}(f), d \Gamma(\omega)\right]=-a^{*}(\omega f)$. Since $\left\|d \Gamma(\omega)^{1 / 2} R_{\omega}^{1 / 2}\right\| \leq 1$, Lemma B.1 implies

$$
\left\|d \Gamma(\omega)^{1 / 2} a^{*}(f) R_{\omega}\right\| \leq\left\|R_{\omega}^{1 / 2} a^{*}(f)\right\|+\left\|R_{\omega}^{1 / 2} a^{*}(\omega f)\right\| \leq\|f / \sqrt{\omega}\|+\|\sqrt{\omega} f\|,
$$

which completes the proof.

Lemma B.3. Let $\chi_{\Omega}$ be the characteristic function of some measurable $\Omega \subset \mathbb{R}^{d}$ and let $N_{\Omega}:=d \Gamma\left(\chi_{\Omega}\right)$. Then, for any $f, g \in L^{2}\left(\mathbb{R}^{d}\right)$,

$$
\left\|\left(1+N_{\Omega}\right)^{-1 / 2} a\left(f \chi_{\Omega}\right) a\left(g \chi_{\Omega}\right)(1+d \Gamma(\omega))^{-1 / 2}\right\| \leq\left\|f \chi_{\Omega} \omega^{-1 / 4}\right\|\left\|g \chi_{\Omega} \omega^{-1 / 4}\right\| .
$$

Proof. Let $\mathcal{F}(\Omega)$ and $\mathcal{F}\left(\Omega^{c}\right)$ denote the symmetric Fock spaces over $L^{2}(\Omega)$ and $L^{2}\left(\Omega^{c}\right)$, respectively, and let $\eta$ denote the (normalized) vacuum in any of these Fock spaces. Let 
$U$ denote the unitary transformation $U: \mathcal{F}\left(\mathbb{R}^{d}\right) \rightarrow \mathcal{F}(\Omega) \otimes \mathcal{F}\left(\Omega^{c}\right)$ defined by $U \eta=\eta \otimes \eta$ and

$$
U a(h) U^{*}=a\left(h \chi_{\Omega}\right) \otimes \mathbb{1}+\mathbb{1} \otimes a\left(h \chi_{\Omega^{c}}\right) .
$$

Then

$$
\begin{aligned}
U d \Gamma(\omega) U^{*} & =d \Gamma\left(\omega \chi_{\Omega}\right) \otimes \mathbb{1}+\mathbb{1} \otimes d \Gamma\left(\omega \chi_{\Omega^{c}}\right), \\
U N_{\Omega} U^{*} & =N \otimes \mathbb{1},
\end{aligned}
$$

where $N$ is the number operator on $\mathcal{F}(\Omega)$ and $h \in L^{2}\left(\mathbb{R}^{d}\right)$. Conjugating by $U$ the operator whose norm is to be estimated, we find that

$$
\begin{aligned}
& \left\|\left(1+N_{\Omega}\right)^{-1 / 2} a\left(f \chi_{\Omega}\right) a\left(g \chi_{\Omega}\right)(1+d \Gamma(\omega))^{-1 / 2}\right\| \\
& =\|\left((1+N)^{-1 / 2} \otimes \mathbb{1}\right)\left(a\left(f \chi_{\Omega}\right) \otimes \mathbb{1}\right)\left(a\left(g \chi_{\Omega}\right) \otimes \mathbb{1}\right) \\
& \quad\left(\mathbb{1} \otimes \mathbb{1}+d \Gamma\left(\omega \chi_{\Omega}\right) \otimes \mathbb{1}+\mathbb{1} \otimes d \Gamma\left(\omega \chi_{\Omega^{c}}\right)\right)^{-1 / 2} \| \\
& \leq\left\|(1+N)^{-1 / 2} a\left(f \chi_{\Omega}\right) a\left(g \chi_{\Omega}\right)\left(1+d \Gamma\left(\omega \chi_{\Omega}\right)\right)^{-1 / 2}\right\|_{\mathcal{F}(\Omega)},
\end{aligned}
$$

where the index of the norm indicates the space on which the operator acts. Following Nelson in the proof of Lemma 5 of [6], we obtain for $\Psi \in D\left(d \Gamma(\omega)^{1 / 2}\right) \cap \mathcal{F}(\Omega)$

$$
\left\|(1+N)^{-1 / 2} a\left(f \chi_{\Omega}\right) a\left(g \chi_{\Omega}\right) \Psi\right\|_{\mathcal{F}(\Omega)} \leq\left\|f \chi_{\Omega} \omega^{-1 / 4}\right\|\left\|g \chi_{\Omega} \omega^{-1 / 4}\right\|\left\|d \Gamma(\omega)^{1 / 2} \Psi\right\|_{\mathcal{F}(\Omega)},
$$

which, combined with (30), proves the lemma.

Lemma B.4. Let $p:=-i \nabla_{x}$, and let $B: L^{2}\left(\mathbb{R}^{d}, d x\right) \rightarrow L^{2}\left(\mathbb{R}^{d}, d x\right) \otimes L^{2}\left(\mathbb{R}^{d}, d k\right)$ denote an operator of the form $\varphi(x) \mapsto b(p, k) e^{-i k x} \varphi(x)$ with some measurable function $(p, k) \mapsto$ $b(p, k)$. Then

$$
\begin{aligned}
\|a(B) \Psi\| & \leq C_{1}(b)\left\|d \Gamma(\omega)^{1 / 2} \Psi\right\|, \\
\left\|a^{*}(B) \Psi\right\| & \leq \max \left\{C_{1}(b), C_{2}(b)\right\}\left\|(1+d \Gamma(\omega))^{1 / 2} \Psi\right\|,
\end{aligned}
$$

where

$$
C_{1}(b):=\left(\sup _{p \in \mathbb{R}^{d}} \int d k \frac{|b(p-k, k)|^{2}}{\omega(k)}\right)^{1 / 2}, \quad C_{2}(b):=\left(\sup _{p \in \mathbb{R}^{d}} \int d k|b(p-k, k)|^{2}\right)^{1 / 2} .
$$

Proof. As in the proof of Lemma B.1, we see that $\|a(B) \Psi\| \leq\left\|B \omega^{-1 / 2}\right\|\left\|d \Gamma(\omega)^{1 / 2} \Psi\right\|$ but now $\left\|B \omega^{-1 / 2}\right\|$ denotes the norm of the operator $B \omega^{-1 / 2}$. Using $\left(B \omega^{-1 / 2} \varphi\right)(p, k)=$ $b(p, k) \hat{\varphi}(p+k)$ and making the substitution $p+k \rightarrow k$ one easily finds that $\left\|B \omega^{-1 / 2}\right\| \leq$ $C_{1}(b)$ which proves the first estimate.

The proof of the second estimate is more involved: By normal ordering

$$
\begin{aligned}
\left\|a^{*}(B) \Psi\right\|^{2} & =\int d k \int d k^{\prime}\left\langle B(k) a_{k}^{*} \Psi, B\left(k^{\prime}\right) a_{k^{\prime}}^{*} \Psi\right\rangle \\
& =\int d k\|B(k) \Psi\|^{2}+\int d k \int d k^{\prime}\left\langle B(k) a_{k^{\prime}} \Psi, B\left(k^{\prime}\right) a_{k} \Psi\right\rangle,
\end{aligned}
$$


where

$$
\begin{aligned}
\int d k\|B(k) \Psi\|^{2} & =\int|b(p, k)|^{2}\|\Psi(p+k)\|^{2} d k d p \\
& =\int|b(p-k, k)|^{2}\|\Psi(p)\|^{2} d k d p \\
& \leq\left(\sup _{p \in \mathbb{R}^{d}} \int d k|b(p-k, k)|^{2}\right)\|\Psi\|^{2} .
\end{aligned}
$$

Let $e_{k}$ denote the operator of multiplication with $e^{-i k x}$ in the particle position space. Then $B(k)=b(p, k) e_{k}=e_{k} b(p-k, k)$. For the integrand of the second term of (31), we therefore have

$$
\begin{aligned}
& \left\langle B(k) a_{k^{\prime}} \Psi, B\left(k^{\prime}\right) a_{k} \Psi\right\rangle=\left\langle b(p, k) e_{k} a_{k^{\prime}} \Psi, b\left(p, k^{\prime}\right) e_{k^{\prime}} a_{k} \Psi\right\rangle \\
& =\left\langle b\left(p-k-k^{\prime}, k\right) e_{k^{\prime}}^{*} a_{k^{\prime}} \Psi, b\left(p-k-k^{\prime}, k^{\prime}\right) e_{k}^{*} a_{k} \Psi\right\rangle \\
& =\int d p\left\langle b\left(p-k-k^{\prime}, k\right) a_{k^{\prime}} \Psi\left(p-k^{\prime}\right), b\left(p-k-k^{\prime}, k^{\prime}\right) a_{k} \Psi(p-k)\right\rangle \\
& \leq \int d p\left|b\left(p-k-k^{\prime}, k\right)\right|\left\|a_{k^{\prime}} \Psi\left(p-k^{\prime}\right)\right\| \cdot\left|b\left(p-k-k^{\prime}, k^{\prime}\right)\right|\left\|a_{k} \Psi(p-k)\right\| \\
& =\int d p \frac{\left|b\left(p-k-k^{\prime}, k\right)\right|}{\sqrt{\omega(k)}} \sqrt{\omega\left(k^{\prime}\right)}\left\|a_{k^{\prime}} \Psi\left(p-k^{\prime}\right)\right\| \frac{\left|b\left(p-k-k^{\prime}, k^{\prime}\right)\right|}{\sqrt{\omega\left(k^{\prime}\right)}} \sqrt{\omega(k)}\left\|a_{k} \Psi(p-k)\right\|
\end{aligned}
$$

The integrand here is of the form $f\left(p, k, k^{\prime}\right) f\left(p, k^{\prime}, k\right)$. Using that $f\left(p, k, k^{\prime}\right) f\left(p, k^{\prime}, k\right) \leq$ $\frac{1}{2}\left(f\left(p, k, k^{\prime}\right)^{2}+f\left(p, k^{\prime}, k\right)^{2}\right)$ and integrating with respect to $k$ and $k^{\prime}$, we arrive at the bound

$$
\begin{aligned}
& \int d k \int d k^{\prime} \int d p \frac{\left|b\left(p-k-k^{\prime}, k^{\prime}\right)\right|}{\omega\left(k^{\prime}\right)} \omega(k)\left\|a_{k} \Psi(p-k)\right\|^{2} \\
& \leq \sup _{p \in \mathbb{R}^{d}} \int d k \frac{|b(p-k, k)|^{2}}{\omega(k)}\left\|d \Gamma(\omega)^{1 / 2} \Psi\right\|^{2}
\end{aligned}
$$

for the second term of (31). This completes the proof.

Lemma B.5. Let $p:=-i \nabla_{x}$ and $F_{x}(k):=f(k) e^{-i k x}$ with $|k|^{s} f \in L^{2}\left(\mathbb{R}^{d}\right)$ for some $s \in[0,1]$. Then, for all $\Psi \in D\left(d \Gamma(\omega)^{1 / 2}\right)$,

$$
\begin{aligned}
\left\|a\left(\left[|p|^{s}, F\right]\right) \Psi\right\| & \leq\left\||k|^{s} f \omega^{-1 / 2}\right\|\left\|d \Gamma(\omega)^{1 / 2} \Psi\right\| \\
\left\|a^{*}\left(\left[|p|^{s}, F\right]\right) \Psi\right\| & \leq \max \left\{\left\||k|^{s} f\right\|,\left\||k|^{s} f \omega^{-1 / 2}\right\|\right\} \quad\left\|(1+d \Gamma(\omega))^{1 / 2} \Psi\right\| .
\end{aligned}
$$

Proof. Note that

$$
\left[|p|^{s}, F_{x}(k)\right]=|p|^{s} f(k) e^{-i k x}-f(k) e^{-i k x}|p|^{s}=\left(|p|^{s}-|p+k|^{s}\right) f(k) e^{-i k x}
$$

and ||$p \pm\left. k\right|^{s}-\left.|p|^{s}|\leq| k\right|^{s}$ for all $p, k \in \mathbb{R}^{d}$ and $s \in[0,1]$. The Lemma now follows by Lemma B.4. 
Lemma B.6. Let $p:=-i \nabla_{x}, \ell \in \mathbb{R}^{d}$, and $F_{x}(k):=f(k) e^{-i k x}$ with $|k|^{s / 2} f \in L^{2}\left(\mathbb{R}^{d}\right)$ for some $s \in[0,1]$. Then, for all $\Psi \in D\left(d \Gamma(\omega)^{1 / 2}\right)$,

$$
\begin{aligned}
& \left\|a\left(\left[|p \pm \ell|^{s}-|p|^{s}, F\right]\right) \Psi\right\| \leq 2|\ell|^{s / 2}\left\||k|^{s / 2} f \omega^{-1 / 2}\right\|\left\|d \Gamma(\omega)^{1 / 2} \Psi\right\|, \\
& \left\|a^{*}\left(\left[|p \pm \ell|^{s}-|p|^{s}, F\right]\right) \Psi\right\| \leq 2|\ell|^{s / 2} \max \left\{\left\||k|^{s / 2} f\right\|,\left\||k|^{s / 2} f \omega^{-1 / 2}\right\|\right\}\left\|(d \Gamma(\omega)+1)^{1 / 2} \Psi\right\| .
\end{aligned}
$$

Proof. We have

$$
\left[|p \pm \ell|^{s}-|p|^{s}, F_{x}(k)\right]=D_{s}(p, k, \ell) f(k) e^{-i k x}
$$

with

$$
D_{s}(p, k, \ell):=|p \pm \ell|^{s}-|p|^{s}-|p+k \pm \ell|^{s}+|p+k|^{s} .
$$

For $p, k, \ell \in \mathbb{R}^{d}$ and $s \in[0,1]$,

$$
\left|D_{s}(p, k, \ell)\right| \leq 2|k|^{s} \quad \text { and } \quad\left|D_{s}(p, k, \ell)\right| \leq 2|\ell|^{s} .
$$

That implies that

$$
\left|D_{s}(p, k, \ell)\right| \leq 2 \min \left\{|k|^{s},|\ell|^{s}\right\}=2 \min \left\{|k|^{s / 2},|\ell|^{s / 2}\right\}^{2} \leq 2|k|^{s / 2}|\ell|^{s / 2} .
$$

This upper bound is independent of the first argument $p$. The lemma now follows from Lemma B.4.

\section{Mapping properties of Weyl operators}

This appendix collects some important identities and estimates in connection with the Weyl operator $e^{i \pi(f)}$ and the dressing transform of Gross. The first lemma recalls a well-known fact, see e.g. Proposition 5.2.4.(1) in Bratteli and Robinson [2]. The second lemma generalizes Lemma B.4 of [4]. As in main part of this paper, we will always assume an admissible $\omega$, which means that $\omega \in L_{l o c}^{\infty}\left(\mathbb{R}^{d}\right)$ and that $\omega>0$ almost everywhere.

Lemma C.1. Let $f, g \in L^{2}\left(\mathbb{R}^{d}\right)$. Then $e^{i \pi(f)} D(\phi(g))=D(\phi(g))$ and

$$
e^{i \pi(f)} \phi(g) e^{-i \pi(f)}=\phi(g)+2 \operatorname{Re}\langle g, f\rangle \quad \text { on } D(\phi(g)) \text {. }
$$

Lemma C.2. Let $f, g \in L^{2}\left(\mathbb{R}^{d}\right)$. Then

$$
\left.\|\left(e^{i \pi(f)}-e^{i \pi(g)}\right)(1+d \Gamma(\omega))\right)^{-1 / 2} \| \leq 2 \max \left\{\|f-g\|,\left\|(f-g) \omega^{-1 / 2}\right\|\right\}+|\operatorname{Im}\langle f, g\rangle| .
$$

Proof. Let $\Psi \in D\left(d \Gamma(\omega)^{1 / 2}\right)$. In analogy to the proof of Lemma B.4 of [4], we get

$$
\left\|\left(e^{i \pi(f)}-e^{i \pi(g)}\right) \Psi\right\| \leq\|\pi(f-g) \Psi\|+|\operatorname{Im}\langle g, f\rangle|\|\Psi\|
$$

By Lemma B.1, we obtain

$$
\|\pi(f-g) \Psi\| \leq 2 \max \left\{\|f-g\|,\left\|\frac{f-g}{\omega^{1 / 2}}\right\|\right\}\left\|(1+d \Gamma(\omega))^{1 / 2} \Psi\right\|
$$

which completes the proof. 
Lemma C.3. Let $f \in L^{2}\left(\mathbb{R}^{d}\right)$ with $\omega f \in L^{2}\left(\mathbb{R}^{d}\right)$. Then $e^{i \pi(f)} D(d \Gamma(\omega))=D(d \Gamma(\omega))$ and on $D(d \Gamma(\omega))$

$$
e^{i \pi(f)} d \Gamma(\omega) e^{-i \pi(f)}=d \Gamma(\omega)+\phi(\omega f)+\left\|\omega^{1 / 2} f\right\|^{2} .
$$

Proof. For $\Psi \in D(d \Gamma(\omega)) \cap \mathcal{F}_{0}$ we have

$$
e^{i \pi(f)} \Psi=\sum_{n \geq 0} \frac{i^{n}}{n !} \pi(f)^{n} \Psi
$$

where $\pi(f)^{n} \Psi \in D(d \Gamma(\omega))$ because $\omega f \in L^{2}\left(\mathbb{R}^{d}\right)$. By a straightforward computation

$$
\begin{aligned}
d \Gamma(\omega) i^{n} \pi(f)^{n} \Psi=i^{n} \pi(f)^{n} d \Gamma(\omega) \Psi & -n i^{n-1} \pi(f)^{n-1} \phi(\omega f) \Psi \\
& +n(n-1) i^{n-2} \pi(f)^{n-2}\left\|\omega^{1 / 2} f\right\|^{2} \Psi
\end{aligned}
$$

and hence

$$
\sum_{n \geq 0} \frac{i^{n}}{n !} d \Gamma(\omega) \pi(f)^{n} \Psi=e^{i \pi(f)}\left(d \Gamma(\omega)-\phi(\omega f)+\left\|\omega^{1 / 2} f\right\|^{2}\right) \Psi .
$$

Since $d \Gamma(\omega)$ is a closed operator, it follows that $e^{i \pi(f)} \Psi \in D(d \Gamma(\omega))$ and that the equation of the lemma holds for the chosen $\Psi$. These results now extend to all $\Psi \in D(d \Gamma(\omega))$ because $D(d \Gamma(\omega)) \cap \mathcal{F}_{0}$ is an operator core and because of the inequality $\left\|d \Gamma(\omega) e^{i \pi(f)} \Psi\right\| \leq C_{\omega, f}(\|d \Gamma(\omega) \Psi\|+\|\Psi\|)$, which follow from the transformation formula of the lemma on this core and from Lemma B.1. Since the sign of $f$ was immaterial in the above arguments, we have shown that $e^{i \pi( \pm f)} D(d \Gamma(\omega)) \subset D(d \Gamma(\omega))$. This implies that $e^{i \pi(f)} D(d \Gamma(\omega))=D(d \Gamma(\omega))$.

The next lemma is not an immediate corollary of the previous one, because of the relaxed assumption on $f$.

Lemma C.4. Suppose $f \in L^{2}\left(\mathbb{R}^{d}\right)$ with $\omega^{1 / 2} f \in L^{2}\left(\mathbb{R}^{d}\right)$. Then, $e^{i \pi(f)} D\left(d \Gamma(\omega)^{1 / 2}\right)=$ $D\left(d \Gamma(\omega)^{1 / 2}\right)$, and for all $\Psi \in D\left(d \Gamma(\omega)^{1 / 2}\right)$,

$$
\left\|d \Gamma(\omega)^{1 / 2} e^{i \pi(f)} \Psi\right\| \leq\left\|d \Gamma(\omega)^{1 / 2} \Psi\right\|+\left\|\omega^{1 / 2} f\right\|\|\Psi\| .
$$

Proof. Let $\Psi \in D(d \Gamma(\omega))$ and define $\omega_{\varepsilon}:=\omega(1+\varepsilon \omega)^{-1}$ for $\varepsilon \geq 0$. Then $\omega_{\varepsilon} f \in L^{2}\left(\mathbb{R}^{d}\right)$, $\omega_{\varepsilon}(k)>0$ almost everywhere, and hence, by Lemma C.3.

$$
\begin{aligned}
\left\|d \Gamma\left(\omega_{\varepsilon}\right)^{1 / 2} e^{i \pi(f)} \Psi\right\|^{2} & =\left\langle\Psi, e^{-i \pi(f)} d \Gamma\left(\omega_{\varepsilon}\right) e^{i \pi(f)} \Psi\right\rangle \\
& =\left\langle\Psi,\left(d \Gamma\left(\omega_{\varepsilon}\right)-\phi\left(\omega_{\varepsilon} f\right)+\left\|\omega_{\varepsilon}^{1 / 2} f\right\|^{2}\right) \Psi\right\rangle \\
& \leq\left\|d \Gamma\left(\omega_{\varepsilon}\right)^{1 / 2} \Psi\right\|^{2}+2\|\Psi\|\left\|a\left(\omega_{\varepsilon} f\right) \Psi\right\|+\left\|\omega_{\varepsilon}^{1 / 2} f\right\|^{2}\|\Psi\|^{2} \\
& \leq\left(\left\|d \Gamma\left(\omega_{\varepsilon}\right)^{1 / 2} \Psi\right\|+\left\|\omega_{\varepsilon}^{1 / 2} f\right\|\|\Psi\|\right)^{2}
\end{aligned}
$$

where Lemma B.1 with $\alpha=1 / 2$ was used in the last inequality. Letting $\varepsilon \rightarrow 0$ the desired inequality is obtained for $\Psi \in D(d \Gamma(\omega))$. Since $D(d \Gamma(\omega))$ is a form core, this inequality extends to all $\Psi \in D\left(d \Gamma(\omega)^{1 / 2}\right)$ and then it proves that $e^{i \pi(f)} D\left(d \Gamma(\omega)^{1 / 2}\right) \subset$ $D\left(d \Gamma(\omega)^{1 / 2}\right)$ provided that $\left\|\omega^{1 / 2} f\right\|<\infty$. Since we may replace $f$ by $-f$ in this proof, the converse inclusion holds as well. 
Corollary C.5. Let $f \in L^{2}\left(\mathbb{R}^{d}\right)$ with $\omega^{1 / 2} f \in L^{2}\left(\mathbb{R}^{d}\right)$. Then

$$
\begin{aligned}
& \left\|(1+d \Gamma(\omega))^{1 / 2} e^{i \pi(f)}(1+d \Gamma(\omega))^{-1 / 2}\right\| \leq 1+\left\|\omega^{1 / 2} f\right\|, \\
& \left\|(1+d \Gamma(\omega))^{-1 / 2} e^{i \pi(f)}(1+d \Gamma(\omega))^{1 / 2}\right\| \leq 1+\left\|\omega^{1 / 2} f\right\| .
\end{aligned}
$$

Proof. By a computation very similar to the one in the proof of Lemma C.4, for all $\Psi \in D\left(d \Gamma(\omega)^{1 / 2}\right)$,

$$
\left\|(1+d \Gamma(\omega))^{1 / 2} e^{i \pi(f)} \Psi\right\| \leq\left\|(1+d \Gamma(\omega))^{1 / 2} \Psi\right\|+\left\|\omega^{1 / 2} f\right\|\|\Psi\| .
$$

This implies the first estimate of the corollary. The second one follows from the first, as it concerns the adjoint operator upon replacing $f$ with $-f$.

The next two lemmas are needed in Section 2 and they generalize statements (a) and (b) of Lemma 3.1 in [4. Recall, that $D\left(H_{0}\right)=D\left(p^{2}\right) \cap D(d \Gamma(\omega))$ and $D\left(H_{0}^{1 / 2}\right)=$ $D(|p|) \cap D\left(d \Gamma(\omega)^{1 / 2}\right)$.

Lemma C.6. Let $p:=-i \nabla_{x}$ and $F_{x}(k):=f(k) e^{-i k x}$ with $f, \omega^{1 / 2} f, k f, k f \omega^{-1 / 2} \in$ $L^{2}\left(\mathbb{R}^{d}\right)$. Then $e^{i \pi(F)} D\left(H_{0}^{1 / 2}\right)=D\left(H_{0}^{1 / 2}\right)$ and

$$
e^{i \pi(F)} p e^{-i \pi(F)}=p-\phi(k F)-\langle f, k f\rangle \quad \text { on } D\left(H_{0}^{1 / 2}\right) .
$$

Proof. Following the proof of Lemma 3.1(a) in [4], we define $\mathscr{D}:=D\left(H_{0}\right) \cap \mathscr{H}_{0}$, which is an operator core and hence a form core of $H_{0}$. For $\Psi \in \mathscr{D}$ one shows that

$$
p e^{-i \pi(F)} \Psi=e^{-i \pi(F)}(p-\phi(k F)-\langle f, k f\rangle) \Psi
$$

by expanding $e^{-i \pi(F)}$ in its exponential series. Here we used that $[\phi(k F), \pi(F)]=$ $2 i\langle f, k f\rangle$, which commutes with $\pi(F)$. Since $\mathscr{D}$ is a form core of $H_{0}$ and since $(p-$ $\phi(k F)-\langle f, k f\rangle)$ is bounded with respect to $H_{0}^{1 / 2}$, by Lemma B.1, Equation (32) extends to all $\Psi \in D\left(H_{0}^{1 / 2}\right)$ and we see that $e^{-i \pi(F)} D\left(H_{0}^{1 / 2}\right) \subset D(|p|)$. Since $D\left(H_{0}^{1 / 2}\right)=$ $D(|p|) \cap D\left(d \Gamma(\omega)^{1 / 2}\right)$ and since $D\left(d \Gamma(\omega)^{1 / 2}\right)$ is left invariant by $e^{-i \pi(F)}$ because of the assumption $\omega^{1 / 2} f \in L^{2}\left(\mathbb{R}^{d}\right)$, see Lemma C.4 we conclude that $e^{-i \pi(F)} D\left(H_{0}^{1 / 2}\right) \subset$ $D\left(H_{0}^{1 / 2}\right)$. Likewise, $e^{i \pi(F)} D\left(H_{0}^{1 / 2}\right) \subset D\left(H_{0}^{1 / 2}\right)$ by changing the sign of $f$ and the lemma is proved.

The condition $\langle f, k f\rangle=0$ in the following lemma simplifies the identity (32), established by Lemma C.6, but it is otherwise inessential.

Lemma C.7. Let $p:=-i \nabla_{x}$ and $F_{x}(k):=f(k) e^{-i k x}$ with $f \in L^{2}\left(\mathbb{R}^{d}\right)$ such that $\omega f, k^{2} f, f \omega^{-1 / 2}, k^{2} f \omega^{-1 / 2} \in L^{2}\left(\mathbb{R}^{d}\right)$ and $\langle f, k f\rangle=0$. Then, $e^{i \pi(F)} D\left(H_{0}\right)=D\left(H_{0}\right)$ and

$$
e^{i \pi(F)} p^{2} e^{-i \pi(F)}=(p-\phi(k F))^{2} \quad \text { on } D\left(H_{0}\right) .
$$

Proof. Let $\Psi \in D\left(H_{0}\right) \subset D\left(H_{0}^{1 / 2}\right)$. Then, by Lemma C. 6 , $e^{-i \pi(F)} \Psi \in D\left(H_{0}^{1 / 2}\right)$ and

$$
p e^{-i \pi(F)} \Psi=e^{-i \pi(F)}(p-\phi(k F)) \Psi .
$$


Since $\Psi \in D\left(H_{0}\right)$, it is clear that $p \Psi \in D(|p|)$. By Lemma B.1 and standard estimates,

$$
\begin{aligned}
\|p \phi(k F)\| \leq & \left\|\phi\left(k^{2} F\right) \Psi\right\|+\|\phi(k F) p \Psi\| \\
\leq & 2 \max \left\{\left\|k^{2} f\right\|,\left\|k^{2} f \omega^{-1 / 2}\right\|\right\}\left\|(1+d \Gamma(\omega))^{1 / 2} \Psi\right\| \\
& +2 \max \left\{\|k f\|,\left\|k f \omega^{-1 / 2}\right\|\right\}\left\|(1+d \Gamma(\omega))^{1 / 2} p \Psi\right\|,
\end{aligned}
$$

where

$$
\left\|(1+d \Gamma(\omega))^{1 / 2} p \Psi\right\| \leq\|(1+d \Gamma(\omega)) \Psi\|+\left\|p^{2} \Psi\right\|,
$$

which shows that $\phi(k F) \Psi \in D(|p|)$ as well, by the assumptions on $f$. Thus, $(p-$ $\phi(k F)) \Psi \in D(|p|)$.

From (33) it also follows that $p \Psi \in D\left(d \Gamma(\omega)^{1 / 2}\right)$ and from Lemma B.2 that

$$
\left\|d \Gamma(\omega)^{1 / 2} \phi(k F) \Psi\right\| \leq 4 \max \left\{\left\|\omega^{1 / 2} k f\right\|,\|k f\|,\left\|k f \omega^{-1 / 2}\right\|\right\}(\|d \Gamma(\omega) \Psi\|+\|\Psi\|)
$$

such that $\phi(k F) \Psi \in D\left(d \Gamma(\omega)^{1 / 2}\right)$ as well, by the assumptions on $f$. Therefore, $(p-$ $\phi(k F)) \Psi \in D(|p|) \cap D\left(d \Gamma(\omega)^{1 / 2}\right)=D\left(H_{0}^{1 / 2}\right)$, such that, using Lemma C.6 again, $e^{-i \pi(F)}(p-\phi(k F)) \Psi \in D\left(H_{0}^{1 / 2}\right)$ and we can apply $p$ on this state again. This leads to

$$
p^{2} e^{-i \pi(F)} \Psi=e^{-i \pi(F)}(p-\phi(k F))^{2} \Psi
$$

and means also that $e^{-i \pi(F)} \Psi \in D\left(p^{2}\right)$. From Lemma C.3, we already know that $e^{-i \pi(F)} D\left(H_{0}\right) \subset D(d \Gamma(\omega))$ such that we conclude that $e^{-i \pi(F)} D\left(H_{0}\right) \subset D\left(H_{0}\right)$. Likewise, $e^{i \pi(F)} D\left(H_{0}\right) \subset D\left(H_{0}\right)$ by changing the sign of $f$ and the lemma is proved.

\section{Acknowledgement}

The authors thank Jacob Schach Møller for many discussions on this and related projects in the course of the past years. A.W. also had a useful discussion with Stefan Teufel that inspired our proof of Theorem 4.5, and he had many discussions with Martin Könenberg on technical issues. The work of Andreas Wünsch was supported by the Deutsche Forschungsgemeinschaft (DFG) through the Research Training Group 1838: Spectral Theory and Dynamics of Quantum Systems.

\section{References}

[1] Zied Ammari. Asymptotic completeness for a renormalized nonrelativistic Hamiltonian in quantum field theory: the Nelson model. Math. Phys. Anal. Geom., $3(3): 217-285,2000$.

[2] Ola Bratteli and Derek W. Robinson. Operator algebras and quantum statistical mechanics. 2. Texts and Monographs in Physics. Springer-Verlag, Berlin, second edition, 1997. Equilibrium states. Models in quantum statistical mechanics.

[3] Jean Ginibre, Fabio Nironi, and Giorgio Velo. Partially classical limit of the Nelson model. Ann. Henri Poincaré, 7(1):21-43, 2006. 
[4] Marcel Griesemer and Andreas Wünsch. Self-adjointness and domain of the Fröhlich Hamiltonian. J. Math. Phys., 57(2):021902, 15, 2016.

[5] Masao Hirokawa, Fumio Hiroshima, and Herbert Spohn. Ground state for point particles interacting through a massless scalar Bose field. Adv. Math., 191(2):339$392,2005$.

[6] Edward Nelson. Interaction of nonrelativistic particles with a quantized scalar field. J. Mathematical Phys., 5:1190-1197, 1964.

[7] Andreas Wünsch. Self-Adjointness and Domain of a Class of Generalized Nelson Models. dissertation, Universität Stuttgart, 2017. 